\title{
Considerations and Challenges for Sex-Aware Drug Repurposing
}

Jennifer L. Fisher ${ }^{1}$. Emma F. Jones ${ }^{1}$. Victoria L. Flanary ${ }^{1}$. Avery S. Williams ${ }^{1}$. Elizabeth J. Ramsey ${ }^{1}$. Brittany N. Lasseigne ${ }^{1}$.

1. Department of Cell, Developmental and Integrative Biology, Heersink School of Medicine, University of Alabama at Birmingham, Birmingham, 35294, USA

Dr. Brittany N. Lasseigne is the corresponding author (bnp0001@uab.edu).

\section{Abstract}

Sex differences are essential factors in disease etiology and manifestation in many diseases such as cardiovascular disease, cancer, and neurodegeneration (1). The biological influence of sex differences (including genomic, epigenetic, hormonal, immunological, and metabolic differences between males and females) and the lack of biomedical studies considering sex differences in their study design has led to several policies. For example, the National Institute of Health's (NIH) sex as a biological variable (SABV) and Sex and Gender Equity in Research (SAGER)) policies to motivate researchers to consider sex differences (2). However, drug repurposing, a promising alternative to traditional drug discovery by identifying novel uses for FDA-approved drugs, lacks sex-aware methods that can improve the identification of drugs that have sex-specific responses (1,3-5). Sex-aware drug repurposing methods either select drug candidates that are more efficacious in one sex or deprioritize drug candidates based on if they are predicted to cause a sex-bias adverse event (SBAE), unintended therapeutic effects that are more likely to occur in one sex. Computational drug repurposing methods are encouraging approaches to develop for sex-aware drug repurposing because they can prioritize sex-specific drug candidates or SBAEs at lower cost and time than traditional drug discovery. Sex-aware methods currently exist for clinical, genomic, and transcriptomic information $(3,6,7)$. They have not expanded to other data types, such as DNA variation, which has been beneficial in other drug repurposing methods that do not consider sex (8). Additionally, some sex-aware methods suffer from poorer performance because a disproportionate number of male and female samples are available to train computational methods (3). However, there is development potential for several different categories (i.e., data mining, ligand binding predictions, molecular associations, and networks). Low-dimensional representations of molecular association and network approaches are also especially promising candidates for future sex-aware drug repurposing methodologies because they reduce the multiple hypothesis testing burden and capture sex-specific variation better than the other methods $(9,10)$. Here we review how sex influences drug response, the current state of drug repurposing including with respect to sex-bias drug response, and how model organism study design choices influence drug repurposing validation.

\section{Keywords}

sex differences, drug repurposing, sex-bias, sex-aware, review, therapeutics, pharmaceuticals, computational drug repurposing 


\section{Highlights}

- Genetic, epigenetic, hormonal, immunological, metabolic, and environmental factors affect sex-biased drug responses.

- Drug repurposing approaches provide a significant advantage over novel drug development by reducing lengthy and costly clinical trials.

- Advances in compute processing power and optimized algorithms for computational systems have increased the efficiency and feasibility of computational drug repurposing.

- Multiple challenges still need to be addressed for sex-aware drug repurposing, including the insufficient understanding of the cause of variation of drug responses due to sex differences, better performing sex-aware repurposing methods, and the lack of large and balanced datasets to develop improved methods.

- Future low-dimensional representations of molecular association and network approaches could significantly impact the field of sex-aware drug repurposing.

\section{Introduction}

Attempting to isolate novel therapeutic drug candidates can cost 1 to 2 billion dollars and 12-16 years of research (7). As an alternative, drug repurposing strategies require less investment and lead to faster Food and Drug Administration (FDA) approval because repurposed candidates are already FDA-approved for alternative indications. Historically, drug repurposing has been serendipitous (7). For example, hydroxychloroquine was initially approved only to treat malaria and later repurposed to treat other autoimmune diseases such as systemic lupus erythematosus (SLE). This repurposing resulted from retrospective clinical studies that found patients with SLE had better outcomes when treated with hydroxychloroquine for other conditions besides SLE (11). Another fortuitous drug repurposing example, sildenafil, was initially intended for ischemic chest pain. However, after phase I clinical trials, it was repurposed to treat erectile dysfunction because of the unintended therapeutic effect reported (7). Recently, through advancements in computational approaches, drug repurposing has become more systematic in predicting drug candidates that are effective and avoid adverse events (7). This review will discuss the following drug repurposing categories and how they apply to sex-aware drug repurposing: data mining, ligand-target binding prediction, molecular associations, and network computational drug repurposing.

The effects of sex differences are known to lead to variation in therapeutic outcomes. For example, tumor resection followed by radiation and treatment with temozolomide is the standard treatment for Glioblastoma Multiforme (GBM) and is more efficacious in females (12). This might be because females are more likely to have the DNA repair enzyme O6-methylguanine-DNA methyltransferase (MGMT) promoter methylated, a biomarker for a therapeutic response for temozolomide (13). Another example of a sex-bias drug response is ibuprofen. This over-the-counter medication is more effective in males than females, even though no pharmacokinetic differences between the sexes have been identified (14). However, pain receptors and nociception differences connected to estrogen activity in the nervous system 
might cause this variation in drug response (15). Even though several examples of sex-bias drug responses exist, most drug repurposing methods do not consider sex. In addition, the available sex-aware drug repurposing methods require algorithmic improvements (e.g., potentially incorporating additional data types) to achieve better performance characteristics in order to improve sex-aware drug repurposing (3).

Adverse events, defined by the FDA as any undesirable experience using a medical product in a patient (16), are the fourth leading cause of death in the United States and can be caused by many factors such as tissue differences, age, development, and race $(3,17)$. However, in 2001, eight of the ten drugs recalled by the FDA were more significant health risks to women than men (18). This recall led to several studies that found that women are twice as likely to experience an adverse drug event compared to men based on adverse drug event case reports from the Food Drug Administration's Adverse Event Reporting System (FAERS) or World Health Organization's VigiBase database $(3,5,19,20)$. Recently, during the coronavirus disease of 2019 (COVID-19) pandemic, there was an increase in the sex-bias adverse event (SBAEs) gap between females and males, possibly due to the pandemic exacerbating known SBAEs such as anxiety (17). Even though SBAEs are more common in females, males are more likely to have a severe drug adverse event than females (20). For instance, ranitidine (an antihistamine and antacid) causes duodenal damage in males (4). Currently, there are several drug repurposing methods to identify drugs that might cause adverse events $(21,22)$. Still, most methods that identify SBAEs, such as AwareDX, have significant limitations and would benefit from improvements to their accuracy $(3,5,23-25)$.

To summarize, sex differences influence drug safety and efficacy, but drug repurposing, as a field, rarely considers sex differences when selecting or prioritizing drug candidates. In this review, we discuss biological mechanisms causing sex-dependent drug responses. In addition, we summarize current drug repurposing methods, survey cases where it has been done, and consider the challenges of developing and evaluating new drug repurposing candidates in light of sex.

\section{Mechanisms Driving Sex-Dependent Variation in Drug Response}

In this review, we describe the current challenges and progress in the field of sex-aware drug repurposing by reviewing variations in drug response due to sex differences (Figure 1). Here sex refers to the $X X$ (female) or $X Y$ (male) genotype of an individual and is the focus of this review. Intersex and genotypes other than $X X$ or $X Y$, have not been extensively studied through future study is necessary and warranted. Gender refers to the societal construct of roles for women and men, which do not always overlap with biological sex (26). Thus, we use sex to refer to a person's genotype and gender to refer to a person's social behavior. Sex differences can be sexually dimorphic, meaning a gene or phenotype is present in one sex but not the other, or sex-biased, meaning there are differences in effect or effect size between the sexes. In this review, we use sex differences and sex bias interchangeably. 
Aside from environmental and social factors, almost all documented molecular sex differences arise from differences in the sex chromosomes, where mammalian females have two copies of the $X$ chromosome and males have one $X$ and one $Y$ chromosome. This basic genetic difference leads to changes in gene expression that give way to larger-scale phenotypic changes as an organism continues to develop. During fetal development, the SRY gene on the $Y$ chromosome codes for "maleness", becoming especially apparent when dysfunction of the SRY gene leads to XY-genotyped individuals developing female sex characteristics (27). However, sex-biased genes are not limited to sex chromosomes and can originate from autosomes (28). When an organism has reached adulthood, many sex differences in gene expression are tissue-specific, with a large amount of differentially-expressed genes in tissues such as adipose, liver, and breast; but this can vary depending on the population of cell types in a given tissue (28-30). In addition, multiple biological pathways have sex-biased gene expression and transcriptional regulation, including sex-biased expression quantitative trait loci (eQTLs) (28). Sex-biased gene expression has been associated with sex-biased diseases, and they are more likely to be drug targets of FDA-approved drugs than non-sex-biased genes (31).

In addition to genetic differences, there are also epigenetic disparities between males and females, specifically in DNA methylation and histone acetylation and methylation $(32,33)$. For example, DNA CpG island methylation is strongly associated with X-chromosome inactivation $(\mathrm{XCl})$, which controls gene dosage compensation and has sex-specific patterns (34). Lyonization (i.e., $\mathrm{XCl}$ ) randomly inactivates either parental or maternal $\mathrm{X}$ chromosome copy resulting in tissue mosaicism, differential expression of parentally imprinted genes, and increased expression for genes that escape $\mathrm{XCl}$ (35). About $15 \%$ of genes on the inactive $X$ chromosome are consistently still expressed, and an additional 10\% variably escape inactivation. This results in a 'double dosage', which leads to a higher level of gene expression in females $(36,37)$. Tissue mosaicism and inheritance of both parental imprints of the $X$ chromosome, as opposed to only the maternal imprint (38), may protect females from deleterious alleles (1). For histone modifications, neonatal male and female mice brains have been found to be sexually dimorphic in histone $\mathrm{H} 3$ Lys9 acetylation (H3K9/14Ac) and trimethylation (H3K9me3) (33). Additionally, there is documentation of sex-differentiated distribution of epigenetic marks such as histone H3 Lys27 trimethylation (H3K27me3), which is also associated with heterochromatic gene repression and X-chromosome inactivation (28). Thus, these differences should be considered when identifying novel cancer drugs, many of which are epigenetic inhibitors that target DNA methyltransferases, histone deacetylases (HDACs), and histone methyltransferases (39). Epigenetic modifiers have the potential to disrupt female dosage compensation, and the chemotherapy HDAC-inhibitor drug vorinostat has been shown to affect levels of H3K27me3 (39).

Many epigenetic changes that occur early in development are mediated by hormones. It is well established that males and females have differences in sex steroid hormones such as testosterone, estrogen, and progesterone (40). These hormones vary in production site, blood concentration, and organ interactions (41). For example, males produce testosterone in the testes and produce more testosterone than estrogen, while females produce more estrogen and progesterone. These hormones, with many others, are necessary for reproduction (42). Sex 
hormones modulate body mass and fluids, enzyme synthesis, synthesis of triglycerides and high-density lipoprotein, and glucose metabolism (43), all of which can affect drug processing. Through steroid response elements (44) and G-coupled protein receptors (45) sex hormones affect gene expression, intracellular signaling, and downstream drug processing. Estrogen receptors, an example of steroid response elements, have an impact on energy intake and expenditure, regulation of adipose tissue distribution, insulin sensitivity, and the function of macrophages and immune cells (46). These sex hormone signaling mechanisms may lead to downstream sex differences in endocytosis of drug transporters, therefore affecting drug response (25).

Sex chromosome genes and sex hormones, in addition to environmental and age-related factors, heavily influence immune responses (47). Females have a higher antibody response, increased amounts of immunoglobulin, and a larger frequency of B cells than males, which leads to their ability to have a more robust immune response (47). $\mathrm{XCl}$ may be one of the main influences of increased immune response in females because the inactive $X$ chromosome can become reactivated in lymphocytes, resulting in the overexpression of autoimmune genes (48). These sex differences in immune responses lead to differential susceptibility to autoimmune diseases, which disproportionately affect females $(49,50)$, and certain cancers, which disproportionately affect males (47). The observed discrepancy in autoimmunity could, in part, be due to sex differences in the microbiome affecting sex hormone regulation (51). These immune system inconsistencies may explain different pharmacokinetic responses to vaccines and various immunologic drugs (52).

There are many differences in all stages of pharmacokinetics between males and females including drug absorption, distribution, metabolism, and elimination (53). Some of the contributing factors to differential drug absorption rates are variations in gastric enzymes, transporter proteins, and liver and kidney organ function (54). For example, females have a higher gastric $\mathrm{pH}$ than men, which can increase the absorption of compounds such as caffeine through decreased ionization of weak bases (54). In terms of the distribution of a drug throughout the body, plasma volume, body mass index (BMI), average organ blood flow, total body water versus body fat, and cardiac output all have sex differences (54). Since females have a higher fat composition than males, the volume of distribution differs depending on whether a drug is lipid-soluble or water-soluble. In this scenario, a water-soluble drug would have a higher volume of distribution in males than in females, and vice versa for lipid-soluble drugs (53). A higher volume of distribution results in higher concentration, so drug dosages should compensate for these effects to avoid the risk of adverse side effects. Pregnancy can cause changes in the elimination half-life of drugs, so the dosage requirements for drugs also need to be adjusted for pregnant individuals (55).

The drug zolpidem, also known as Ambien, is a drug the FDA recommended to be given to females at a dose half that of males; however, this recommendation only increased the adjusted dosage compliance from $10 \%$ to $15 \%$ (56). The FDA zolpidem statement was due to pharmacokinetic and pharmacodynamic differences causing lower rates of clearance in women, resulting in $40 \%-50 \%$ higher concentrations of the drug and a higher likelihood of side effects 
such as extreme drowsiness, possibly from non-compliance with the FDA dosage recommendations (57). Zolpidem is among many drugs affected by the myriad of differences in drug metabolism in the cytochrome p450 (CYP) enzymes. Sex differences in the CYP superfamily of genes, which are involved with phase I drug metabolism, can explain some discrepancies in pharmacokinetic processes between males and females (58). For example, differences in CYP genes such as CYP1A2, CYP2B6, CYP2E1, CYP3A4, affect the metabolism of hundreds of compounds (59). CYP2B6 has more than 70 substrates (including ketamine), and females have higher overall activity compared to males (59). CYP3A4 is involved in the metabolism of over $50 \%$ of all drugs (including zolpidem), and females have been found to have $20 \%-50 \%$ higher activity than males (59). In addition, the CYP superfamily of genes is involved in sex hormone biosynthesis (60).

Finally, differing environmental and social pressures can lead to conscious decisions that physically affect the body, resulting in differing responses to various drugs. These social pressures vary depending on gender, not just biological sex. Gendered behaviors may lead to changes in testosterone levels in men and women (61). One example of environmental and social contributions is melanoma: men are more likely than women to develop melanoma and have a fatal outcome (62). The increased likelihood of development could be due to many behavioral differences: men spend more time outside, are less likely to wear sunscreen, and are less likely to self-detect and examine for skin irregularities (63). Males and females have different immune responses, so women's higher melanoma survival rate could be due to women's immune systems being more effective at preventing metastasis through estrogen signaling (62). Women are also more likely to use supplements, natural botanicals, and homeopathic remedies, which are less likely to be reported than FDA-approved drugs and could cause dangerous drug interactions $(64,65)$. There are also nutritional and gut microbiome differences between males and females (66). These microbiome differences can be caused by various factors, including hormones, diet, drugs, BMI, and colonic transit time (66). Acetaminophen is an over-the-counter drug known to have different toxicity across individuals, which may be due to microbial metabolites that compete with acetaminophen for liver enzyme binding sites (67). This competition leads to a higher fraction of acetaminophen transformed into a toxic byproduct resulting in increased hepatotoxicity (67).

In substance use, men in the United States are more likely to smoke cigarettes than women (68). Incomplete combustion leads to accumulations of carcinogenic compounds, which are inducers of CYP enzymes (69), causing many drug interactions, with some drugs requiring dosage increases due to higher CYP1A2 levels (70). Among biological females who are heavy cigarette smokers, low-dose oral contraceptives have a much higher chance of adverse arterial effects (71); therefore, the FDA advised doctors not to prescribe oral contraceptives for females smoking over 15 cigarettes a day (72). Another example of how behavior can lead to biological consequences is the example of alcohol, which interacts with numerous drugs (73). Alcohol has a stronger effect in women due to differences in alcohol metabolism, leading to higher amounts of alcohol in the body and a higher risk for severe side effects and adverse drug reactions (54). Women are drinking at increased rates on a population scale, and because of their 
predisposition to stronger effects of alcohol, they are at higher risk for alcohol-related health problems such as liver disease than men $(74,75)$.

Due to the overwhelming evidence that males and females have differing responses to many drugs, their treatment recommendations should reflect these discrepancies. Therefore, the need to develop alternative drug treatments to minimize sex-bias related adverse side effects is a high priority, and drug repurposing can help address this in a more timely and cost-effective manner.

\section{Overview of Drug Repurposing}

As drug discovery costs increase (145\% between 2003 and 2013), the need to use systematic methods to identify drug repurposing candidates has grown $(76,77)$. Some of these approaches are experimental while others are computational. Experimental approaches conduct drug screens using in vitro and in vivo models testing hundreds to thousands of compounds and evaluating if those compounds affect a specific molecular target or cellular phenotype (7). An example of a large-scale application of a drug screen is the Profiling Relative Inhibition Simultaneously in Mixtures (PRISM) project (78). This project treated 930 cancer cell lines with 21,000 drugs to identify which inhibit cancer growth (78). This large-scale screening process requires many resources including cell lines, drugs, and personnel time and expertise. However, researchers can reduce this investment by identifying specific drug candidates via computational approaches that will prioritize candidates for experimental application.

Over the last several decades, increased processing power and optimized algorithms for rapid calculations have resulted in in silico drug repurposing methods being quicker at identifying drug repurposing candidates than exhaustive experimental approaches (Table 1) (7). Additionally, the number and size of biomedical databases with appropriate clinical, genomic, transcriptomic, epigenetic, metabolic, and proteomic information for various diseases and preclinical models have expanded (79-81). These public databases have expedited the process of identifying new drug candidates by making this data accessible to research groups across the world to train their drug repurposing approaches (79-81). Here we discuss data mining, ligand-target binding prediction, molecular associations, and network computational drug repurposing categories that contain different strategies for identifying drug repurposing candidates.

Data mining drug repurposing approaches retrospectively analyze information from and across clinical trials, biomedical literature, and other resources with drug outcome or drug target information to identify novel drug indications (77). These approaches apply machine learning models that use logical and mathematical algorithms to interpret or make predictions about data. For example, Kuenzi et al. generated an interpretable visible neural network, a machine learning model, to predict the effectiveness of drugs for individual cancer mutation profiles (8). Another example of a data mining approach, text mining, uses biomedical literature to connect information from different studies or data sources to discover novel connections or patterns (82). For example, aspirin, an over-the-counter medication used for analgesia, was repurposed in 2016 to reduce the risk of developing colorectal cancer after a systematic review of data from 
the literature and clinical trials (83). An advantage of these data mining approaches is using large amounts of publicly available data that researchers do not need to recreate for themselves. However, one limitation of data mining approaches is the dependency on information from literature and clinical trials. If a disease or drug is rare or understudied, there may be limited publications for these approaches.

Another drug repurposing approach, ligand-target binding prediction, identifies drugs predicted to bind to a disease target (i.e., proteins) based on their binding affinity (84). Molecular dynamic modeling and structure similarity are two types of ligand-target binding prediction methods. Molecular docking predicts if a ligand and a drug target can bind via their structures (84). A limitation of this approach is that it requires a significant amount of time and memory, even on a high-performance computing system (85). Alternatively, structure similarity approaches predict drug candidates based on the premise that similar drugs will have similar mechanisms of action or adverse events (86). A general limitation of ligand-target binding prediction is the requirement for accurate information about drug structures, mechanism of actions, and protein structures of disease targets to predict suitable drug candidates, but these are often inaccurate or unknown (7).

Molecular association methods identify targets or patterns from molecular data (i.e., genomic, transcriptomic, epigenetic, metabolic, or proteomic profiles) that correlate with disease, therapeutic outcomes, and/or drug candidates (7). Molecular association strategies include guilt-by-association, signature matching, and signature reversion (7). Recently, a genome-wide association study (GWAS) used guilt-by-association to identify drug repurposing candidates for psoriasis (87). In this study, IL-23 receptor gene variants were found to be associated with the development of psoriasis, and therefore the IL-23 receptor became a potential drug target (87). Further, based on biomedical literature, risankizumab was identified as a drug candidate because it targets the IL-23 receptor, and after clinical trials, risankizumab was indeed approved for psoriasis treatment $(87,88)$. Signature matching, another molecular association strategy, has been applied in several cancer applications, as reviewed in Wang et al. (88). It compares patient molecular profiles to cell line profiles or another model system that were treated with drugs and assessed for a specific phenotype (e.g.., cell viability in cancer cell lines) (88). Another variation, signature reversion, leverages molecular disease signatures (i.e., gene expression differences between disease and normal) and cell line perturbation signatures (i.e., gene expression differences before and after drug treatment) to identify drug signatures that are inversely related to disease signatures (81). Chen et al. applied this principle to liver cancer and identified and validated four drug repurposing candidates in xenograft mouse models (89). Signature matching and reversion can be approached by either enrichment statistics such as Kolmogorov-Smirnov or correlation methods (90). However, enrichment statistics approaches had lower accuracy compared to correlation approaches, but correlation approaches were more sensitive to noise $(90,91)$. Additionally, molecular associations methods can only determine correlation and not causation; therefore, molecular associations are not always the drug target or the cause of the disease. For example, if a GWAS study identified a gene variant with favored drug response, it should not conclude that the gene with the variant or gene closest to the variant is the drug target. A neighboring gene could be the drug target because of the influence of linkage 
disequilibrium where genes near each other tend to be inherited together (7). Therefore, researchers using these molecular association strategies should be critical when evaluating and interpreting their associations to avoid making causal inferences about drug targets (7).

Network approaches evaluate mathematical graphs (nodes joined together by edges) representing relationships as edges between different nodes like genes, proteins, diseases, and drugs to identify drug repurposing candidates (92). One of the benefits of this approach is that networks can integrate multiple data types to predict drug candidates. In one study, Morselli et al. successfully repurposed four drug repurposing candidates for COVID-19 by implementing a protein-protein interaction network, information about severe acute respiratory syndrome coronavirus 2 (SARS-CoV-2), and drug targets (93). Another benefit of a network approach is interpretability. This interpretability allows for insights into possible disease and drug mechanisms. For example, a study that used tissue-specific networks derived from transcription factor sequence motifs, protein-protein interaction, and gene expression data identified the BTK inhibitor ibrutinib as a drug candidate for metabolic syndrome (94). Because of the interpretability of their network approach, the researchers gained mechanistic insight into how ibrutinib treatment might treat metabolic syndrome via BTK expression and immune cells (94). However, some drug-target interaction networks with nodes representing drugs and gene targets have a high number of false positives due to nonspecific drug targets making it difficult for this approach to predict new drug candidates (95). Another limitation is the dynamic nature of biological systems means networks capture a specific point in time so critical evaluation and interpretation of network construction is necessary (95). Additionally, network analysis can require costly computing resources and time due to complex algorithms (96).

However, a promising approach is to use a combination of computational and experimental approaches to identify and validate drug repurposing candidates (Table 1). For example, Fang, et al. conducted a study for Alzheimer's Disease (AD) with data mining approaches and a drug-target network to identify a drug repurposing candidate, sildenafil, followed by experimental assays to validate its mechanism of action in patient-derived induced pluripotent stem cells (iPSCs) (97). This study used multiple data sources including health insurance claims from the MarketScan Medicare Claims database and gene expression data from the Gene Expression Omnibus (GEO) and Genotype-Tissue Expression (GTEx) databases (97). Moreover, some studies combine several methods (known as weak learners) into a single framework, resulting in better therapeutic predictions than using one method alone (90). This technique is called an ensemble approach. EMUDRA, an example of an ensemble method, combined four weak learners: Kolmogorov-Smirnov statistic, weighted signed statistic, the sum of fold changes, and cosine similarity (90). This ensemble model outperformed the individual weak learners and other drug repurposing approaches with simulated and drug perturbation data (90).

Although many computational methods have prioritized novel drug repurposing candidates, they still have many limitations. A common hurdle for all of these methods is the requirement for statistically powered datasets to create more accurate models. This limitation can be incredibly challenging for sex-aware drug repurposing because these methods require powered datasets for males and females. In combination with other challenges to studying sex differences 
(discussed in the last section), these limitations make sex-aware drug repurposing difficult. Still, we propose and discuss several solutions and drug repurposing approaches to mitigate these challenges to aid in the development of better sex-aware drug repurposing approaches (discussed in the next section).

\section{Sex-Aware Drug Repurposing Methods}

Currently, there are limited methods available to either select sex-specific drug repurposing candidates that will be effective against a disease of interest or prioritize candidates to avoid SBAEs. Here we summarize the currently available sex-aware approaches or studies for sex-aware drug repurposing (Table 2).

The first sex-aware drug repurposing approaches use data mining to identify SBAEs based on case information from patient adverse events databases such as FAERS or VigiBase. A study from Yu et al. calculated the reporting odds ratio for a sex-bias adverse event based on FAERS' case reports (5). They found and confirmed several SBAEs and drug combinations via drug labels or previous studies (5). The drug repurposing database DrugCentral also used the FAERS database to calculate the likelihood ratio for a sex-bias adverse event for all drugs in the database. These calculations can identify drugs with SBAEs and prioritize drug candidates that avoid SBAEs (98). Another study created a random forest model based on the FAERS database to predict a propensity score (the likelihood that a patient is female) based on clinical data and the standardized medical terminology used for medical conditions, medicines, and medical devices (Medical Dictionary for Regulatory Activities MedDRA) adverse events terms(3). This study used several metrics such as out-of-bag score and Receiver Operating Characteristic Area Under the Curve (ROC-AUC) to evaluate their random forest model. This model had an out-of-bag score of 0.63 and ROC-AUC of 0.64 (3). However, this model had a low recall of 0.47 and required 250 patients per sex for each adverse event (3). A more recent study from Zucker and Prendergast conducted a literature search to identify SABEs based on pharmacokinetic differences between males and females (4). This approach was successful in identifying female-bias adverse events but struggled to identify male-bias adverse events (4). This limitation might suggest pharmacokinetics are less likely to cause male-biased adverse events (4). In addition, this study was limited by only having pharmacokinetic information for a small fraction of FDA-approved drugs (4).

Furthermore, there are some additional limitations with data mining studies. For example, adverse events are often voluntarily reported by healthcare professionals, consumers, and drug manufacturers (99). Even with standardized terminology like MedDRA, it can be difficult to categorize or describe an adverse event; therefore, there could be misclassification of a specific adverse event. Second, case reports cannot distinguish an adverse event caused by a drug or an extraneous factor such as another drug. Some databases like FAERS do not require a causal relationship for a report to be filed (99). Therefore, it is difficult to determine if a drug causes an adverse event. However, it is possible to estimate disproportionality or calculate odds ratios to identify associations between drugs and adverse events (17). 
Next, we identified two sex-aware molecular association studies that used molecular biomarkers to identify sex-specific drug candidates or SBAEs. The first, a study by Kfoury et al., identified drug repositioning candidates not currently FDA approved for any condition that might benefit GBM patients (12). This group previously reported that GBM is sexually dimorphic because of the variation in gene expression profiles between males and females that they associated with different survival between the sexes (12). After this study, they hypothesized that the gene expression variation might be due to sex differences in epigenetic regulation (12). Specifically, Kfoury et al. investigated the bromodomain and extraterminal (BET) family of proteins, epigenetic readers of histone lysine acetylation (100). Their study identified that BET inhibitors (JQ1 and RVX208) decreased tumor growth in male tumors but increased the growth in female tumors (100). This study exemplifies how molecular differences between males and females can lead to a hypothesis resulting in the discovery of a drug candidate. Another study analyzed drug perturbation profiles from Connectivity MAP, an extensive drug perturbation by cell line database, to determine what drugs perturbed heart-specific sex-biased genes as determined by differential gene expression analysis (6). With this information about which drugs perturbed heart-specific sex-bias genes, they found sex-bias drug responses for acebutolol, tacrine, and metformin in rat models and further validated their results with clinical information from a human patient cohort (6). Currently, this sex- and tissue-specific approach is limited to heart tissue because they only investigated sex-biased gene expression in heart tissue. However, researchers can adapt this method to develop more tissue-specific and sex-aware models.

One sex-aware experimental approach is the manipulation of sex hormones as a therapy for a disease. Such manipulation is used when one sex tends to respond better to current therapies and/or have better outcomes than the other. For example, in $A D$, which is more prevalent in females compared to males, leuprolide acetate, an androgen deprivation therapy used for the treatment of prostate cancer, has been investigated for the treatment of $A D$ as it might slow the progression of the disease $(101,102)$.

While most of these methods performed poorly due to limited, sex-balanced datasets. Future development of analytical strategies to more accurately model sex-bias from these unbalanced datasets or leverage new and more balanced datasets. Also, we were unable to identify sex-aware methods for network or ligand-binding drug repurposing categories. Therefore, we suggest approaches to improve sex-aware drug repurposing for data mining, ligand-target binding prediction, molecular associations, and network computational drug repurposing categories.

Molecular association and network approaches are promising candidates for future sex-aware drug repurposing methodologies. Molecular association studies provide molecular biomarkers that might be causal for different responses to drugs (6). These methods can separate males and females to identify genes or other biomarkers to determine drug repurposing candidates or prioritize drugs. The GTEx study discovered that gene expression differences between males and females tend to be small (28). An alternate approach to evaluating gene-level sex differences or individual biomarkers would be to develop metagenes, signatures, or low-dimensional representations of gene expression, DNA variation, or protein expression to 
identify drug repurposing candidates. Using low-dimensional representations of molecular patterns reduces the multiple hypothesis testing burden to find significant differences between males and females (9). These differences could be associated with drug response or adverse events.

As an emerging computational approach for sex-aware drug repurposing, network techniques developed by the Network Zoo have been used to build gene regulatory networks to identify regulatory pattern differences between males and females from GTEx tissue samples (10). The authors observed larger sex differences between the edges in the gene regulatory networks than gene expression (10). They also showed sex differences in the regulatory pattern of drug metabolism in colon cancer, indicating a possible sex difference in drug response (23). Another network method developed by the Network Zoo group, ALPACA (ALtered Partitions Across Community Architectures), determined network module sex differences in breast tissues (103). These differences were associated with intracellular estrogen receptor signaling pathways, developmental and signaling pathways, and pathways related to breast cancer (103). While these network approaches did not identify candidates for drug repurposing, sex-specific networks capture variation due to sex differences better than differential gene expression because network approaches identified more significant differences between edges and network communities (i.e., groups of related nodes and edges in networks) in male and female gene regulatory networks compared to differences in gene expression $(10,103)$. Therefore, the use of male and female networks and current network methods is a potential future direction.

Due to several limitations, data mining and ligand-target binding prediction approaches are challenging to adapt for sex-aware drug repurposing. First, data mining approaches typically require large amounts of balanced data, equal male and female data points. However, several databases are not balanced. For example, the UK biobank is more female-biased (as of 2021: 273,000 females \& males 229,00 ) while GTEx is more male-biased ( v8 release: 636 males \& 312 females) $(28,104,105)$. In addition, retroactive studies should consider using downsampling techniques because older clinical trials did not require female subjects, biasing clinical studies toward male subjects (26). For basic biomedical research data, human samples are slightly female-biased (52.1\%) while mice samples are male-biased (62.5\%) (106). Furthermore, this bias varies between different biomedical research disciplines with reproductive studies having more female-only studies while pharmacology has more male-specific studies (107). Also, studies have historically failed to report the sex for their samples (106). Therefore, future studies should carefully consider what datasets are being used and apply methods to overcome disproportional datasets to create sex-aware data mining approaches.

Ligand-target binding prediction methods could also be difficult to adjust for sex differences. Several studies have identified that sex differences can be due to gene regulatory and hormone signaling $(10,28)$. Therefore, ligand-target binding prediction, a method that only evaluates how a ligand and target interacts, does not traditionally consider how other influences such as hormones will affect the ligand-target binding interaction $(10,108)$. This is also a limitation for experimental target binding assays, too. However, one potential avenue is to compare structures of drugs with known sex-bias responses to identify drugs with potential for sex-bias 
drug responses. This sex-aware approach is similar to Vilar et al.'s approach, which compares drugs' structures with known adverse events to identify drugs with potential for adverse events (109). Overall, the current methods are not sufficiently developed for sex-aware drug repurposing, but they have the potential.

\section{Challenges and Proposed Solutions for Model Systems for Sex-Aware Drug Repurposing}

Several challenges exist across basic, translational, and clinical research in assessing sex as a biological variable (SABV) in in vitro, in vivo, and in silico model systems (Figure 2). These challenges impact sex-aware drug repurposing because these models are critical for identifying and validating drug repurposing candidates. Here we discuss the challenges and proposed solutions for these model systems.

The genomic basis of sexual differentiation is a confounding factor for all in vitro, in vivo, and in silico model systems. Sex chromosomes experience lower accuracy than autosomes for genotype arrays because of the homologous regions between the $X$ and $Y$ chromosomes (1). Many previous GWAS studies that determined SNPs from genotyping assays removed sex chromosomes from their analysis (110). This resulted in an underrepresentation of SNPs from sex chromosome regions in analyses (110). Therefore, GWAS studies including sex chromsome SNPS due to improved genotyping arrays and analyses are needed to determine the influence of sex chromosomes. Additionally, several studies have also demonstrated poor mapping quality of sequencing data and bias from homologous regions between the sex chromosomes, reducing the ability to detect sex chromosome DNA variation and gene expression accurately (1). However, approaches are now being used to remedy these issues. For example, XYalign, a bioinformatics framework, can be applied to next-generation sequencing data to appropriately account for the sequence homology between the $X$ and $Y$ chromosome by inferring the sex chromosome ploidy of a sample and remapping the sequencing reads to the sex complement of the sample (111). Another approach creates sex-specific reference genomes for sequencing read alignment (112). This approach has been shown to result in more accurate read alignment for both traditional aligners (i.e., STAR, HISAT) and pseudo-aligners (i.e., Salmon) (112). Correct alignment is important for DNA variants on the sex chromosomes because, for example, the CTPS2 and DLG3 X chromosome genes are known to cause differences in drug response (113). Further, the expression of these genes are correlated to the sensitivity of both platinating agents carboplatin and cisplatin (113).

In vitro cell line models are important to drug repurposing because many drug screens and validation experiments use these models for testing the efficacy and toxicity of drug repurposing candidates. However, some cell lines become "de-sexualized" after losing a Y chromosome, in the case of male cell lines, or the loss of an $X$ chromosome, in the case of female cell lines (114). Additionally, after several passages (the number of times a cell line cultured has been harvested and reseeded), female iPSCs will frequently undergo inactive $X$ erosion from loss of $X I S T$ expression (the long coding RNA that causes $X$ inactivation) and reactivate expression of silenced genes on the inactive $X$ chromosome, a process known as inactive $X$ erosion (115). 
Researchers can validate the sex of in vitro models to make their results more rigorous by considering SABV in cell lines. Overall, this will improve experimental drug repurposing screens' ability to predict and validate effective and safe candidates.

Additionally, genetic and hormonal sex differences are also difficult to account for in vivo animal models as these can vary across organisms (116). While there are several benefits to in vivo models such as the ability to test hypotheses in dynamic biological systems, animal models are still not perfect mimics of human biology. For instance, most animals do not follow the same sex determination as mammals (116). For example, in the Drosophila genus of flies, $\mathrm{XX}, \mathrm{XXY}$, and $X X Y Y$ flies are female; while $X Y$ and $X O$ flies are male (117). In flies, The $Y$ chromosome does not impact sex determination and both $X$ chromosomes remain active (117). Another widely used model organism, C. elegans, has XX hermaphrodites and XO males (117). In addition, zebrafish have several different loci across the genome that determines sex (118). Overall, all of these model organisms have been used in drug screens for drug repurposing candidates, but future studies can further investigate how sex difference for model organisms compare to human sex differences (119-121).

Another challenge for in vivo models is sex differences in human phenotypes might not be present in a given model organism. For example, the longevity between males and females varies significantly between species and human females, similar to yellow baboons, tend to live longer (122). Mice, a common model organism in biomedical research, also demonstrate variations in longevity (123). For some mouse strains, the males live longer (ie., 129S1, NOD.B10, and NZW), but in others the females live longer (ie., B10 and P) (123). However, if mouse strains are pooled together, mice do not show a sex difference in longevity (123). Another consideration is hormone differences between humans and model organisms. One study compared rats, mice, and humans by measuring sex hormone levels at different points in development (124). They found that these sex hormones peak at different developmental stages for each organism (124). Overall, how well model organisms reflect sex differences in humans needs further investigation. For example, a recent study suggested that sex-bias gene expression in the proximal tubule cells of kidney in humans did not match sex-bias gene expression in mouse proximal tubule cells (125). With the kidney being an important organ for drug metabolism, this finding can have a major implication for the modeling of drug responses in mice.

In vivo animal studies have historically excluded female animals from many study designs, resulting in less female data (126). For example, in publicly available gene expression data (RNA-Seq and Microarray), 62.5\% of labeled mice gene expression samples are male (106). Stated reasons for excluding female animal models include the perceived need to account for the estrous cycle in female rodents, increase sample sizes of female subjects for statistical power, and increased time and costs associated with these factors (108,127-129). Recently, studies have reported that hormonal fluctuations in animal models do not necessarily lead to increased variability in results for either of the sexes $(108,127-129)$. Therefore, well-powered studies can be designed with minimal increase in sample sizes (i.e., 14-33\%) that still observe the effects of and interactions between two independent variables (130). Studies can do this by 
employing factorial study designs which utilize a 2-way analysis of variance (ANOVA) to discern outcomes due to sex differences from those that result from experimentation $(126,130-132)$. Another approach to further understand the impact of sex chromosomes compared to hormones in mice models is the use of designed studies with models that can discern the effects of sex chromosomes from those of gonadal hormones. For example, hormonal influences can be minimized by using functionally gonadectomized mice, such as the Sf1 knockout mouse $(133,134)$, sex hormone receptor knockout mouce $(135,136)$, or the four-core genotypes model $(134,137,138)$. In this model, the Sry gene is moved from the $Y$ chromosome to an autosome to generate four genotypes: XXF (XX mice with ovaries), XXM (XX mice with testes), XYF (XY mice with ovaries), and XYM (XY mice with testes) $(134,137,138)$. Not only does this allow for observation of gonadal hormone effects separately from sex chromosome effects, but it also identifies sex chromosome influences on non-gonadal tissues (134). In the future, in vivo studies can incorporate other factors such as development/aging and reproductive events (i.e., puberty, pregnancy, and menopause). These events have hormone fluctuations that can also impact sex-bias transcriptomic regulation and drug responses $(25,139)$.

The biological impact of sex extends also to impacts on in silico modeling of biological systems. The exclusion of female animals in preclinical studies (126) and the low enrollment of female patients in clinical trials $(140,141)$ have led to a decrease in data for female subjects and underpowered statistical results for retrospective analyses desegregated by sex (126). There are also the problems of underreporting (not including the distribution of sex across samples) and pooling (acknowledging that both sexes were used in the study but the study did not analyze data for the impact of sex) $(127,142)$. With the recent implementation of NIH's SABV policy in 2016, underreporting of sex has decreased between 2009 and 2019 from $16 \%$ to $6 \%$ in biomedical research articles (107). However, sample pooling is still common in studies with both male and female samples (42\% in 2019 \& 50\% in 2009) (107). Both underreporting and pooling reduce the reproducibility and transparency of scientific research because it masks biological differences between the sexes $(127,142)$. This leads to data accuracy issues and misinterpretation of the results from the study $(127,142)$. With genomic data, there are ways to infer sex if the study does not report them. Researchers can identify the ploidy of the $X$ chromosome or develop sex marker sequences from sex chromosome nucleotide sequences $(111,143)$. Another method developed by Fylnn et al. identified the sex of a sample by the use of an elastic net machine learning classifier (106). This classifier had an accuracy of $91 \%$ in microarray and $88 \%$ in RNA-seq human gene expression data (106). With sufficiently powered data from both male and female subjects across basic, translational, and clinical research, increased quality data will improve in silico models and thus the precision and efficacy of sex-aware drug repurposing approaches.

\section{Conclusion}

Here we described sex-aware drug repurposing and discussed the challenges and future of sex-aware drug repurposing. Drug repurposing is a valuable method for identifying drug candidates for FDA approval because of its ability to prioritize efficacious drug candidates at a reduced cost compared to traditional drug discovery $(7,76)$. However, various drugs have male 
and female-bias responses and adverse events $(4,54)$. This variation in drug response arises because of various sex differences in genetic, epigenetic, hormonal, immunological, metabolic, and environmental factors (1). Several computational drug repurposing approaches exist or are being developed to identify or prioritize drug candidates for both sexes $(3,5,6,23,31,98,100,103)$. This can lead to improved therapeutic options and prevent adverse events for patients. In addition, these drug candidates could provide novel insights into disease manifestation, progression, and underlying mechanisms. This can be beneficial to understanding and treating diseases, such as in the case of the BET inhibitor for GBM discussed (100). Unfortunately, the validation of these drug candidates is limited by existing preclinical models $(106,127)$. Therefore, in line with NIH policy, future studies should routinely investigate how including sex as a biological variable influences study design.

There is an urgent need to address the following: (1) the lack of balanced data to develop accurate models for sex-aware drug repurposing, (2) the need for a variety of improved sex-aware drug repurposing methods, and (3) the scarcity of studies relating to sex differences and variation in drug response between the sexes. Increased representation of females in biomedical research and clinical trials through balanced sex studies or female-only studies is needed to improve drug repurposing approaches. While some methods are available to overcome limited datasets $(3,5,6,23,31,98,100,103)$, ultimately statistically powered datasets provide more accurate modeling and predictions. In addition, the current methods are inadequate for exploring sex-aware drug repurposing but have performance limitations due to the data types used. Also, while sex-aware methods for data mining and molecular association approaches have been developed, there is a lack of sex-aware drug repurposing approaches that apply ligand-target binding prediction and network methods. The development of novel approaches is crucial for identifying future drug repurposing candidates for both sexes. In addition, the field needs sex-aware drug repurposing approaches for different omics data such as epigenetics, metabolomics, etc., which have been beneficial in other drug repurposing methods that do not consider sex $(8,80)$. The current sex-aware methods are biased towards clinical, genomic, and transcriptomic input data. Lastly, there is a need for more studies that focus on sex differences across all diseases (if the disease occurs in both sexes). Even five years after the US National Institutes of Health required studies to consider sex as a biological variable, there are still many understudied aspects surrounding sex differences and how they affect drug response (107). The information about sex differences could inspire and improve drug repurposing methods in the future.

Upon reflection, one promising sign that sex-aware methods will improve the field of drug repurposing is how tissue-aware drug repurposing has improved the field. While sex is an essential factor in drug response, other factors such as tissue differences, aging, development, race, social, and environmental factors are also important to consider for drug repurposing methods. For example, a study investigated tissue-specific genetic features of drug target genes (i.e., tissue specificity of gene expression, Mendelian association, phenotype, and tissue-level effects of genome-wide associations loci driven by eQTLs, and genetic constraint) (22). They discovered that these tissue-specific features resulted in a 2.6 more significant risk of side effects, and drug development and repurposing studies could use these tissue-specific genetic 
features to help evaluate drugs (22). Overall, the use of tissue-specific gene expression improved drug safety and efficacy predictions in multiple studies $(22,144)$. This indicates that as drug repurposing expands to account for sex differences, drug safety and efficacy predictions will also improve for both sexes.

In conclusion, the development of sex-aware drug repurposing methods is essential but challenging due to the understudied complexity of sex differences. We recognize these challenges for sex-aware drug repurposing, but its potential for biomedical research and patient care outweighs the difficulties. In the future, sex-aware drug repurposing will identify safer and more efficacious drug candidates for both males and females. 


\section{List of abbreviations}

- NIH- National Institute of Health

- SABV- Sex as a biological variable

- SAGER- Sex and Gender Equity in Research

- SBAE- sex-bias adverse event

- FDA- Food and Drug Administration

- SLE- systemic lupus erythematosus

- GBM- glioblastoma

- COVID-19- coronavirus disease of 2019

- MGMT- O6-methylguanine-DNA methyltransferase

- eQTLs- expression quantitative trait loci

- XCl-X-chromosome inactivation

- H3K9/14ac- histone H3 Lys9 acetylation

- H3K9me3- histone H3 Lys9 trimethylation

- H3K27me3- histone H3 Lys27 trimethylation

- HDAC- histone deacetylases

- CYP- cytochrome p450 enzymes

- PRISM- Profiling Relative Inhibition Simultaneously in Mixtures

- GWAS- genome-wide association study

- SARS-CoV-2- severe acute respiratory syndrome coronavirus 2

- ANOVA- analysis of variance

- AD- Alzheimer's Disease

- FAERS- Food and Drug Administration Adverse Event Reporting System

- ROC-AUC- Receiver Operating Characteristic Area Under the Curve

- MedDRA- Medical Dictionary for Regulatory Activities

- GTEx- Genotype-Tissue Expression project

- GEO- Gene Expression Omnibus

- BET- bromodomain and extraterminal proteins

- ALPACA- Altered Partitions Across Community Architectures

- iPSCs -induced pluripotent stem cells

\section{Declarations}

- Ethics approval and consent to participate

- Not applicable

- Consent for publication

- Not applicable

- Availability of data and materials

- Not applicable

- Competing interests

- The authors declare that they have no competing interests

- Funding 
- This work was supported by R03 (OD R03OD030604) (to BNL, JLF also supported), the UAB Lasseigne Lab Start-Up funds (to BNL), UAB's AMC21 scholarship (to JLF), UAB's MSTP (to VLF), UAB's RACE21 scholarship (to ASW), and the UAB Blazer Fellowship (to JLF \& EFJ).

- Acknowledgements

- We would like to thank the following members of the Lasseigne Lab for reviewing this manuscript: Tabea Soelter, Jordan Whitlock, Dr. TC Howton, Anisha Haldar, and Dr. Kasi McPherson.

- Authors' Contributions

- JLF and BNL devised this review and proof outline. JLF organized and wrote the first draft. EFJ was a major contributor to the writing of the Mechanisms Driving Sex-Dependent Variation in Drug Response section of the manuscript. VLF organized and research the Challenges and Proposed Solutions for Model Systems for Sex-Aware Drug Repurposing section. ASW designed and created the figures. EJR created the tables with the drug repurposing information. All the authors read and approved the manuscript. 


\section{Figures}

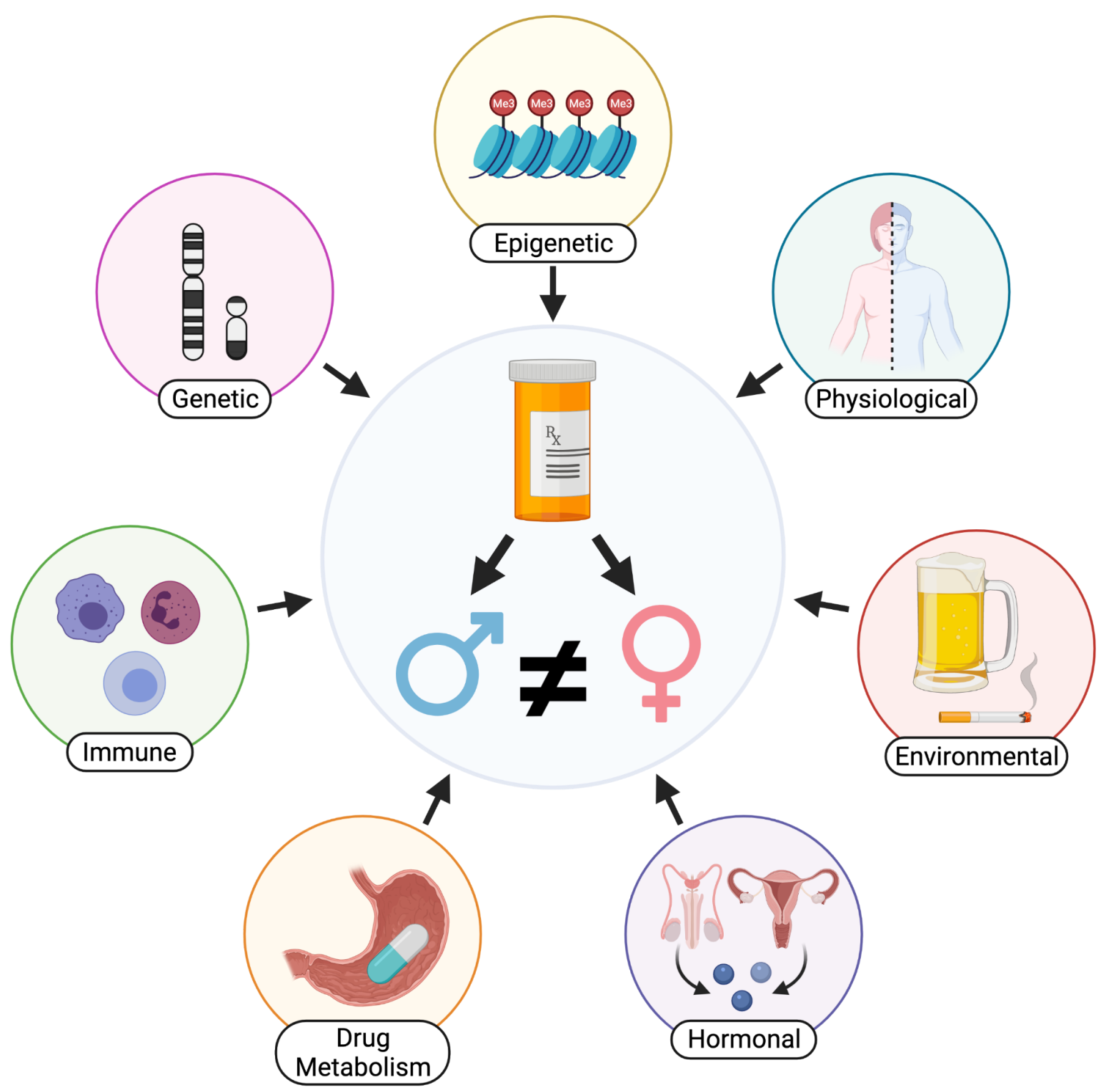

Figure 1: Factors known to influence sex-biased drug response include genetic, epigenetic, hormonal, immunological, metabolic, and environmental factors. 


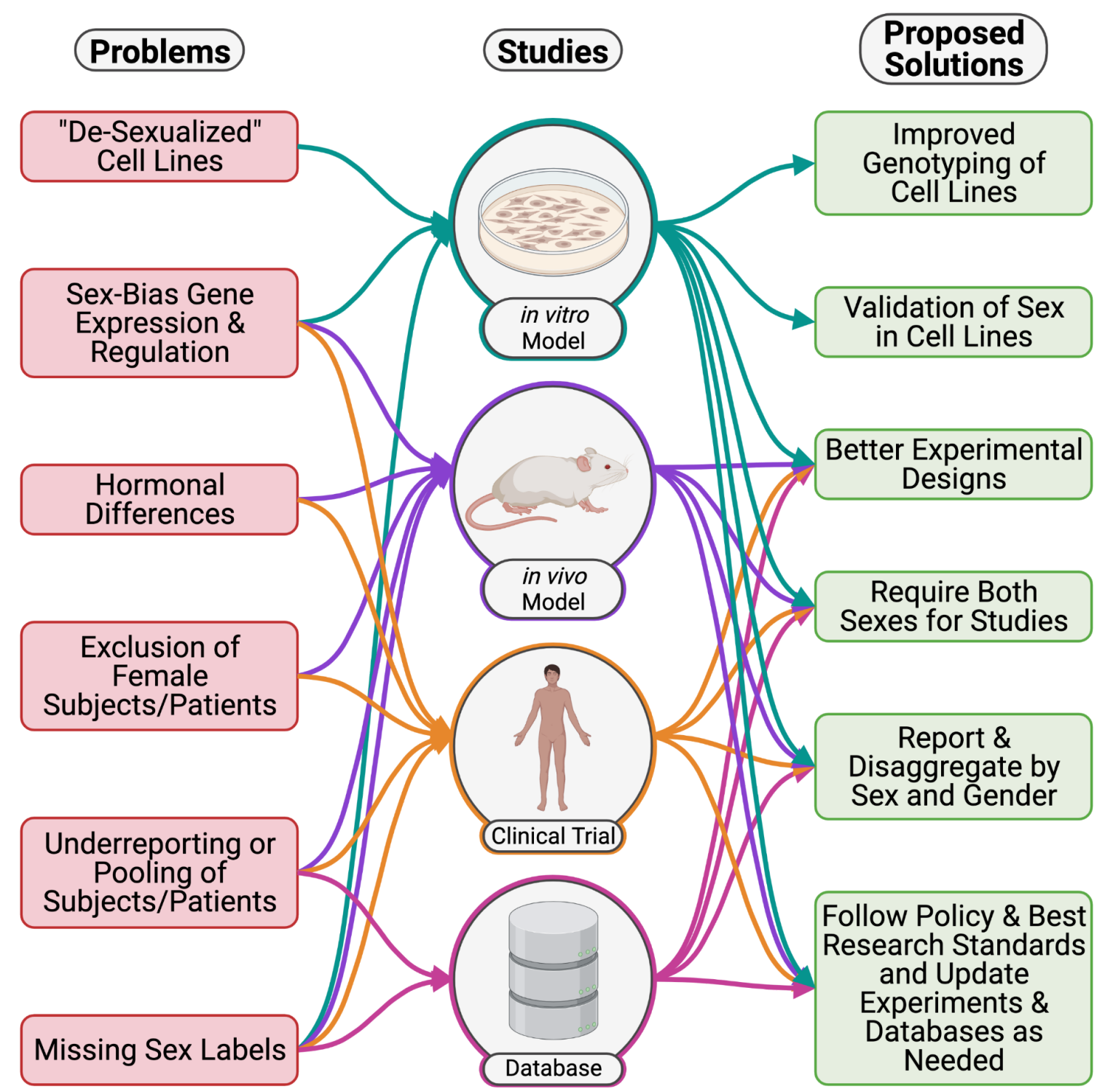

Figure 2: Proposed solutions to sex-aware drug repurposing challenges. Teal arrows are connected to cell lines models. Purple arrows are connected to preclinical models. Orange arrows are connected to clinical trials. Pink arrows are connected to databases. 


\section{Tables}

This table describes the methods of drug repurposing with advantages and disadvantages for each. Examples listed were methods used in studies or by consortiums and research centers.

Table 1: Drug Repurposing Methods Overview

\begin{tabular}{|c|c|c|c|c|}
\hline Method & Description & Advantages & Disadvantages & Examples \\
\hline Data Mining & $\begin{array}{l}\text { Analysis of data from various } \\
\text { sources (including } \\
\text { peer-reviewed published } \\
\text { experimental data, } \\
\text { databases, screens, } \\
\text { pharmaceutical information, } \\
\text { EHR's, etc) }\end{array}$ & $\begin{array}{l}\text { - Crowdsource data } \\
\text { - Multiomic data } \\
\text { accessible } \\
\text { - Reuse of previously } \\
\text { analyzed data }\end{array}$ & $\begin{array}{l}\text { - Limited data for rare } \\
\text { diseases and } \\
\text { understudied drugs, } \\
\text { and dependent on large } \\
\text { sample sizes } \\
\text { - Inconsistency of data } \\
\text { structure } \\
\text { - Ethics/privacy (for } \\
\text { EHR data) }\end{array}$ & $\begin{array}{l}\text { - Mastermind (145) } \\
\text { - Pharos (146) } \\
\text { - Iwata H et al. } 2015 \\
\text { (147) } \\
\text { - Duffy Á et al. } 2020 \\
\text { (122) }\end{array}$ \\
\hline $\begin{array}{l}\text { Ligand-Binding } \\
\text { Prediction }\end{array}$ & $\begin{array}{l}\text { Interactions between ligands } \\
\text { and targets are predicted to } \\
\text { determine suitable } \\
\text { candidates through binding } \\
\text { by structural and chemical } \\
\text { simulation }\end{array}$ & $\begin{array}{l}\text { - Identify novel drug } \\
\text { targets } \\
\text { - Identify novel } \\
\text { compound structures } \\
\text { - Prior knowledge of } \\
\text { protein function not } \\
\text { required } \\
\text { - Detect possible side } \\
\text { effects by off-target } \\
\text { binding }\end{array}$ & $\begin{array}{l}\text { - Requires target's } \\
\text { tertiary structure } \\
\text { - Experimental binding } \\
\text { affinities often not } \\
\text { recapitulated } \\
\text { - Disregards } \\
\text { downstream effects } \\
\text { - Computationally } \\
\text { expensive } \\
\text { - Missing biological } \\
\text { context to allow tissue } \\
\text { or sex-specificity }\end{array}$ & $\begin{array}{l}\text { - Chupakhin V et al. } \\
2013 \text { (148) } \\
\text { - Napolitano F et al. } \\
2013 \text { (149) } \\
\text { - Vilar S et al. } 2014 \\
\text { (150) } \\
\text { - Cao R et al. } 2014 \\
\text { (151) } \\
\text { - Cheng F et al. } \\
2013 \text { (152) }\end{array}$ \\
\hline $\begin{array}{l}\text { Molecular } \\
\text { Associations }\end{array}$ & $\begin{array}{l}\text { Molecular perturbations are } \\
\text { associated with disease, } \\
\text { therapeutic outcomes, or } \\
\text { drug candidates }\end{array}$ & $\begin{array}{l}\text { - Elucidate } \\
\text { drug/disease } \\
\text { mechanisms } \\
\text { - Compatible with } \\
\text { multiomic data } \\
\text { - Detect druggable } \\
\text { pathways } \\
\text { - Exposes off-target } \\
\text { drug effects }\end{array}$ & $\begin{array}{l}\text { - High signal-to-noise } \\
\text { ratio inhibits } \\
\text { deconvolution of } \\
\text { signatures } \\
\text { - Disregards } \\
\text { physiological } \\
\text { interactions } \\
\text { - Associations may not } \\
\text { convey direct } \\
\text { causations }\end{array}$ & $\begin{array}{l}\text { - Dr. Insight (153) } \\
\text { - signatureSearch } \\
(91) \\
\text { - Sanseau P et al. } \\
2012(154) \\
\text { - Grover MP et al. } \\
2015(155)\end{array}$ \\
\hline Networks & $\begin{array}{l}\text { The relationship of genes } \\
\text { within and between } \\
\text { pathways provide insight for } \\
\text { upstream and downstream } \\
\text { drug targets that may infer } \\
\text { treatment for a disease } \\
\text { phenotype and/or show drug } \\
\text { interactions within a } \\
\text { biological system }\end{array}$ & $\begin{array}{l}\text { - Multiomic data } \\
\text { - Reveals relationships } \\
\text { - Determine } \\
\text { mechanistic pathways } \\
\text { - Exposes off-target } \\
\text { drug effects }\end{array}$ & $\begin{array}{l}\text { - Statistically complex } \\
\text { - Computationally } \\
\text { expensive } \\
\text { - Requires strong } \\
\text { signal-to-noise or large } \\
\text { datasets to deconvolute } \\
\text { signal }\end{array}$ & $\begin{array}{l}\text { - Drug2Ways (156) } \\
\text { - Green CS et al. } \\
2015(157)\end{array}$ \\
\hline $\begin{array}{l}\text { Experimental - } \\
\text { Perturbation }\end{array}$ & $\begin{array}{l}\text { Cultured cells are treated } \\
\text { with a variety of drugs and }\end{array}$ & $\begin{array}{l}\text { - Shows gene } \\
\text { expression as a result }\end{array}$ & $\begin{array}{l}\text { - Immortalized cells } \\
\text { - Lacks heterogeneity }\end{array}$ & $\begin{array}{l}\text { - LINCS L1000 } \\
\text { profiles (158) }\end{array}$ \\
\hline
\end{tabular}




\begin{tabular}{|c|c|c|c|c|}
\hline Screens & $\begin{array}{l}\text { screened for phenotypic } \\
\text { response }\end{array}$ & $\begin{array}{l}\text { of perturbation } \\
\text { - Displays consociation } \\
\text { between cell receptors } \\
\text { and pharmaceuticals } \\
\text { - Non-predicted, in-vitro } \\
\text { results }\end{array}$ & $\begin{array}{l}\text { - Limited } \\
\text { microenvironment } \\
\text { - Costly }\end{array}$ & $\begin{array}{l}\text { - Iljin K et al. } 2009 \\
(159) \\
\text { - Shen M et al. } 2018 \\
(160)\end{array}$ \\
\hline $\begin{array}{l}\text { Experimental - } \\
\text { Binding Assays }\end{array}$ & $\begin{array}{l}\text { The chemical engagement of } \\
\text { targets and ligands are } \\
\text { tested in vitro to divulge } \\
\text { repurposed candidates } \\
\text { based on disease-target } \\
\text { matching via affinity/thermal } \\
\text { stabilization and structures }\end{array}$ & $\begin{array}{l}\text { - Physically measured } \\
\text { drug-target binding } \\
\text { activity } \\
\text { - Captures biophysical } \\
\text { features } \\
\text { - Reveals promiscuous } \\
\text { drug-target interactions }\end{array}$ & $\begin{array}{l}\text { - Disregards } \\
\text { downstream effects } \\
\text { - Selection of drugs and } \\
\text { targets are much more } \\
\text { restricted than in silico } \\
\text { approaches due to } \\
\text { feasibility (cost, time, } \\
\text { and accessibility) }\end{array}$ & $\begin{array}{l}\text { - Cellular } \\
\text { ThermoStability } \\
\text { Assay (CETSA) } \\
(161) \\
\text { - Miettinen TP et al. } \\
2014(162)\end{array}$ \\
\hline $\begin{array}{l}\text { Experimental - } \\
\text { Animal Models }\end{array}$ & $\begin{array}{l}\text { Organisms are treated with } \\
\text { drugs to model patient } \\
\text { response and } \\
\text { patient-specific } \\
\text { disease-causing genetic } \\
\text { variants can be introduced to } \\
\text { provide more pertinent } \\
\text { system }\end{array}$ & $\begin{array}{l}\text { - Recapitulates full } \\
\text { physiological system } \\
\text { - Resource for } \\
\text { multiomic data } \\
\text { collection } \\
\text { - In-vivo results } \\
\text { - Patient-specific } \\
\text { models allow for } \\
\text { precision medicine }\end{array}$ & $\begin{array}{l}\text { - Significant financial } \\
\text { and time expense } \\
\text { - Requires } \\
\text { narrowed-down list of } \\
\text { candidates } \\
\text { - Results frequently do } \\
\text { not translate to patient } \\
\text { response } \\
\text { - Orthologous targets } \\
\text { may vary greatly from } \\
\text { human target structure }\end{array}$ & $\begin{array}{l}\text { - UAB C-PAM (139) } \\
\text { - JAX Center for } \\
\text { Precision Genetics } \\
(140) \\
\text { - BCM Center for } \\
\text { Precision Medicine } \\
\text { Models (141) } \\
\text { - vivoChip (142) } \\
\text { - The Hollow Fiber } \\
\text { Model (143) }\end{array}$ \\
\hline
\end{tabular}

Abbreviations: EHR electronic health records

Reporting System, GBM glioblastoma multiforme

Studies, tools, and databases that have taken sex into account for drug repurposing are described here in table 2. The main method is listed (as described in Table 1) as well as examples and a short explanation of how the method integrated sex-specific awareness.

Table 2: Sex-Aware Drug Repurposing Examples.

\begin{tabular}{|l|l|l|l|}
\hline Method & Examples & Development & Sex-Aware Approach \\
\hline Data Mining & Drug Central (98) & Database & $\begin{array}{l}\text { Drug Database compilation using FDA, EMA, and } \\
\text { PMDA; information includes active ingredients, } \\
\text { MOA's, indivations, pharmacological actions, } \\
\text { regulatory data, chemical structure, and adverse } \\
\text { drug events separated by sex to help correct for } \\
\text { sex-bias }\end{array}$ \\
\cline { 2 - 4 } & AwareDX (3) & Study/Analysis & $\begin{array}{l}\text { Pharmacovigilance algorithm that predicts sex-bias } \\
\text { adverse events from FAERS data and found } \\
\text { 20,817 sex-specific drug risks }\end{array}$ \\
\cline { 2 - 4 } & $\begin{array}{l}\text { "Sex differences in } \\
\text { pharmacokinetics predict }\end{array}$ & Study/Analysis & $\begin{array}{l}\text { Pharmacokinetic differences by sex are linked to } \\
\text { sex-specific adverse drug reactions using data }\end{array}$ \\
\hline
\end{tabular}




\begin{tabular}{|c|c|c|c|}
\hline & $\begin{array}{l}\text { adverse drug reactions in } \\
\text { women" ((4)) }\end{array}$ & & procured from ISI Web of Science and PubMed \\
\hline \multirow[t]{3}{*}{$\begin{array}{l}\text { Molecular } \\
\text { Association }\end{array}$} & $\begin{array}{l}\text { "Gender differences in the } \\
\text { effects of cardiovascular } \\
\text { drugs" (24) }\end{array}$ & Study/Analysis & $\begin{array}{l}\text { Sex influences on pharmacokinetics, } \\
\text { pharmacodynamics, and other physiological factors } \\
\text { are reviewed for cardiovascular drug response }\end{array}$ \\
\hline & $\begin{array}{l}\text { "Brd4-bound enhancers drive } \\
\text { cell-intrinsic sex differences in } \\
\text { glioblastoma" (100) }\end{array}$ & Study/Analysis & $\begin{array}{l}\text { Sex-specific epigenetic signatures are identified in } \\
\text { GBM mouse astrocytes and human glioblastoma } \\
\text { stem cells }\end{array}$ \\
\hline & $\begin{array}{l}\text { "Sex-Dependent Gene } \\
\text { Co-Expression in the Human } \\
\text { Body" (31) }\end{array}$ & Study/Analysis & $\begin{array}{l}\text { Across-tissue RNAseq analysis finds co-expression } \\
\text { to be highly sex-dependent }\end{array}$ \\
\hline \multirow[t]{4}{*}{ Networks } & $\begin{array}{l}\text { "Population-scale identification } \\
\text { of differential adverse events } \\
\text { before and during a pandemic" } \\
\text { (17) }\end{array}$ & Study/Analysis & $\begin{array}{l}\text { Sex-specific desparities are presented in network } \\
\text { analysis of adverse drug events before and during } \\
\text { COVID-19 pandemic }\end{array}$ \\
\hline & $\begin{array}{l}\text { "Gene regulatory network } \\
\text { analysis identifies sex-linked } \\
\text { differences in colon cancer } \\
\text { drug metabolism" (23) }\end{array}$ & $\begin{array}{l}\text { Analysis using } \\
\text { PANDA and } \\
\text { LIONESS }\end{array}$ & $\begin{array}{l}\text { Molecular differences investigated using } \\
\text { sex-specific networks to uncover role in metabolism } \\
\text { of drugs in colon cancer }\end{array}$ \\
\hline & $\begin{array}{l}\text { "Sex Differences in Gene } \\
\text { Expression and Regulatory } \\
\text { Networks across } 29 \text { Human } \\
\text { Tissues" (10) }\end{array}$ & $\begin{array}{l}\text { Analysis using } \\
\text { LIONESS }\end{array}$ & $\begin{array}{l}\text { Sex biases are found in patient-specific networks in } \\
\text { every tissue and by disease }\end{array}$ \\
\hline & $\begin{array}{l}\text { "Detecting phenotype-driven } \\
\text { transitions in regulatory } \\
\text { network structure" (103) }\end{array}$ & $\begin{array}{l}\text { Analysis using } \\
\text { ALPACA }\end{array}$ & $\begin{array}{l}\text { Sexual dimorphism are investigated in human } \\
\text { breast tissue gene expression networks }\end{array}$ \\
\hline $\begin{array}{l}\text { Ligand-Binding } \\
\text { Prediction }\end{array}$ & $\begin{array}{l}\text { "3D pharmacophoric similarity } \\
\text { improves multi adverse drug } \\
\text { event identification in } \\
\text { pharmacovigilance" (109) }\end{array}$ & Study/Analysis & $\begin{array}{l}\text { Pharmaceutical 3D structure similarity predictions } \\
\text { are combined with adverse drug events as a } \\
\text { method that may be applied for comparing safety } \\
\text { by sex-aware reporting }\end{array}$ \\
\hline Experimental & $\begin{array}{l}\text { "Sexual differentiation of } \\
\text { central vasopressin and } \\
\text { vasotocin systems in } \\
\text { vertebrates: different } \\
\text { mechanisms, similar } \\
\text { endpoints" (163) }\end{array}$ & Study/Analysis & $\begin{array}{l}\text { Rat model is used in comparison with human } \\
\text { model to compare sex-bias of common } \\
\text { neuropsychiatric drug targets }\end{array}$ \\
\hline
\end{tabular}

Abbreviations: FDA Food and Drug Administration, EMA European Medicines Agency, PMDA Pharmaceuticals and Medical Devices Agency, MOA mechanism of action, FAERS FDA Adverse Event 


\section{References}

1. Khramtsova EA, Davis LK, Stranger BE. The role of sex in the genomics of human complex traits. Nat Rev Genet. 2019 Mar;20(3):173-90.

2. Shansky RM, Murphy AZ. Considering sex as a biological variable will require a global shift in science culture. Nat Neurosci. 2021 Apr;24(4):457-64.

3. Chandak P, Tatonetti NP. Using Machine Learning to Identify Adverse Drug Effects Posing Increased Risk to Women. Patterns (N Y) [Internet]. 2020 Oct 9;1(7). Available from: http://dx.doi.org/10.1016/j.patter.2020.100108

4. Zucker I, Prendergast BJ. Sex differences in pharmacokinetics predict adverse drug reactions in women. Biol Sex Differ. 2020 Jun 5;11(1):32.

5. Yu Y, Chen J, Li D, Wang L, Wang W, Liu H. Systematic Analysis of Adverse Event Reports for Sex Differences in Adverse Drug Events. Sci Rep. 2016 Apr 22;6:24955.

6. Cui C, Huang C, Liu K, Xu G, Yang J, Zhou Y, et al. Large-scale in silico identification of drugs exerting sex-specific effects in the heart. J Transl Med. 2018 Aug 29;16(1):236.

7. Pushpakom S, lorio F, Eyers PA, Escott KJ, Hopper S, Wells A, et al. Drug repurposing: progress, challenges and recommendations. Nat Rev Drug Discov. 2019 Jan;18(1):41-58.

8. Kuenzi BM, Park J, Fong SH, Sanchez KS, Lee J, Kreisberg JF, et al. Predicting Drug Response and Synergy Using a Deep Learning Model of Human Cancer Cells. Cancer Cell. 2020 Nov 9;38(5):672-84.e6.

9. Taroni JN, Grayson PC, Hu Q, Eddy S, Kretzler M, Merkel PA, et al. MultiPLIER: A Transfer Learning Framework for Transcriptomics Reveals Systemic Features of Rare Disease. Cell Syst. 2019 May 22;8(5):380-94.e4.

10. Lopes-Ramos CM, Chen C-Y, Kuijjer ML, Paulson JN, Sonawane AR, Fagny M, et al. Sex Differences in Gene Expression and Regulatory Networks across 29 Human Tissues. Cell Rep. 2020 Jun 23;31(12):107795.

11. Dörner T. Therapy: Hydroxychloroquine in SLE: old drug, new perspectives. Nat Rev Rheumatol. 2010 Jan;6(1):10-1.

12. Yang W, Warrington NM, Taylor SJ, Whitmire P, Carrasco E, Singleton KW, et al. Sex differences in GBM revealed by analysis of patient imaging, transcriptome, and survival data. Sci Transl Med [Internet]. 2019 Jan 2;11(473). Available from:

http://dx.doi.org/10.1126/scitranslmed.aao5253

13. Carrano A, Juarez JJ, Incontri D, Ibarra A, Guerrero Cazares H. Sex-Specific Differences in Glioblastoma. Cells [Internet]. 2021 Jul 14;10(7). Available from:

http://dx.doi.org/10.3390/cells10071783

14. Butcher BE, Carmody JJ. Sex differences in analgesic response to ibuprofen are influenced by expectancy: a randomized, crossover, balanced placebo-designed study. Eur J Pain. 2012 Aug;16(7):1005-13. 
15. Farkouh A, Baumgärtel C, Gottardi R, Hemetsberger M, Czejka M, Kautzky-Willer A. Sex-Related Differences in Drugs with Anti-Inflammatory Properties. J Clin Med Res [Internet]. 2021 Apr 1;10(7). Available from: http://dx.doi.org/10.3390/jcm10071441

16. Office of the Commissioner. What is a Serious Adverse Event? [Internet]. [cited 2021 Oct 4]. Available from:

https://www.fda.gov/safety/reporting-serious-problems-fda/what-serious-adverse-event

17. Zhang $X$, Sumathipala $M$, Zitnik M. Population-scale identification of differential adverse events before and during a pandemic. Nature Computational Science. 2021 Oct 5;1-12.

18. Carey JL, Nader N, Chai PR, Carreiro S, Griswold MK, Boyle KL. Drugs and Medical Devices: Adverse Events and the Impact on Women's Health. Clin Ther. 2017 Jan;39(1):10-22.

19. Mayne BT, Bianco-Miotto T, Buckberry S, Breen J, Clifton V, Shoubridge C, et al. Large Scale Gene Expression Meta-Analysis Reveals Tissue-Specific, Sex-Biased Gene Expression in Humans. Front Genet. 2016 Oct 13;7:183.

20. Watson S, Caster O, Rochon PA, den Ruijter H. Reported adverse drug reactions in women and men: Aggregated evidence from globally collected individual case reports during half a century. EClinicalMedicine. 2019 Dec;17:100188.

21. Failli M, Paananen J, Fortino V. ThETA: transcriptome-driven efficacy estimates for gene-based TArget discovery. Bioinformatics. 2020 Aug 15;36(14):4214-6.

22. Duffy Á, Verbanck M, Dobbyn A, Won H-H, Rein JL, Forrest IS, et al. Tissue-specific genetic features inform prediction of drug side effects in clinical trials. Sci Adv [Internet]. 2020 Sep;6(37). Available from: http://dx.doi.org/10.1126/sciadv.abb6242

23. Lopes-Ramos CM, Kuijjer ML, Ogino S, Fuchs CS, DeMeo DL, Glass K, et al. Gene Regulatory Network Analysis Identifies Sex-Linked Differences in Colon Cancer Drug Metabolism. Cancer Res. 2018 Oct 1;78(19):5538-47.

24. Tamargo J, Rosano G, Walther T, Duarte J, Niessner A, Kaski JC, et al. Gender differences in the effects of cardiovascular drugs. Eur Heart J Cardiovasc Pharmacother. $2017 \mathrm{Jul}$ $1 ; 3(3): 163-82$.

25. Moyer AM, Matey ET, Miller VM. Individualized medicine: Sex, hormones, genetics, and adverse drug reactions. Pharmacol Res Perspect. 2019 Dec;7(6):e00541.

26. Mauvais-Jarvis F, Bairey Merz N, Barnes PJ, Brinton RD, Carrero J-J, DeMeo DL, et al. Sex and gender: modifiers of health, disease, and medicine. Lancet. 2020 Aug 22;396(10250):565-82.

27. Massanyi EZ, Dicarlo HN, Migeon CJ, Gearhart JP. Review and management of 46,XY disorders of sex development. J Pediatr Urol. 2013 Jun;9(3):368-79.

28. Oliva M, Muñoz-Aguirre M, Kim-Hellmuth S, Wucher V, Gewirtz ADH, Cotter DJ, et al. The impact of sex on gene expression across human tissues. Science [Internet]. 2020 Sep 11;369(6509). Available from: http://dx.doi.org/10.1126/science.aba3066

29. Arnold AP, Lusis AJ. Understanding the sexome: measuring and reporting sex differences 
in gene systems. Endocrinology. 2012 Jun;153(6):2551-5.

30. Lu T, Mar JC. Investigating transcriptome-wide sex dimorphism by multi-level analysis of single-cell RNA sequencing data in ten mouse cell types. Biol Sex Differ. 2020 Nov 5;11(1):61.

31. Hartman RJG, Mokry M, Pasterkamp G, den Ruijter HM. Sex-dependent gene co-expression in the human body. Sci Rep. 2021 Sep 21;11(1):18758.

32. Sharp AJ, Stathaki E, Migliavacca E, Brahmachary M, Montgomery SB, Dupre Y, et al. DNA methylation profiles of human active and inactive $X$ chromosomes. Genome Res. 2011 Oct;21(10):1592-600.

33. Tsai H-W, Grant PA, Rissman EF. Sex differences in histone modifications in the neonatal mouse brain. Epigenetics. 2009 Jan;4(1):47-53.

34. Duncan CG, Grimm SA, Morgan DL, Bushel PR, Bennett BD, NISC Comparative Sequencing Program, et al. Dosage compensation and DNA methylation landscape of the $X$ chromosome in mouse liver. Sci Rep. 2018 Jul 4;8(1):10138.

35. Arnold AP. A general theory of sexual differentiation. J Neurosci Res. 2017 Jan 2;95(1-2):291-300.

36. Carrel L, Willard HF. X-inactivation profile reveals extensive variability in X-linked gene expression in females. Nature. 2005 Mar 17;434(7031):400-4.

37. Balaton BP, Brown CJ. Escape Artists of the X Chromosome. Trends Genet. 2016 Jun;32(6):348-59.

38. Babak T, DeVeale B, Tsang EK, Zhou Y, Li X, Smith KS, et al. Genetic conflict reflected in tissue-specific maps of genomic imprinting in human and mouse. Nat Genet. 2015 May;47(5):544-9.

39. Laskowski Al, Fanslow DA, Smith ED, Kosak ST. Clinical Epigenetic Therapies Disrupt Sex Chromosome Dosage Compensation in Human Female Cells. Gend Genome. 2018 Jan;2(1):2-7.

40. Southren AL, Tochimoto S, Carmody NC, Isurugi K. Plasma production rates of testosterone in normal adult men and women and in patients with the syndrome of feminizing testes. J Clin Endocrinol Metab. 1965 Nov;25(11):1441-50.

41. Svechnikov K, Söder O. Ontogeny of gonadal sex steroids. Best Pract Res Clin Endocrinol Metab. 2008 Feb;22(1):95-106.

42. Hess RA. Estrogen in the adult male reproductive tract: a review. Reprod Biol Endocrinol. 2003 Jul 9;1:52.

43. Lauretta R, Sansone M, Sansone A, Romanelli F, Appetecchia M. Gender in Endocrine Diseases: Role of Sex Gonadal Hormones. Int J Endocrinol. 2018 Oct 21;2018:4847376.

44. Nilsson S, Mäkelä S, Treuter E, Tujague M, Thomsen J, Andersson G, et al. Mechanisms of estrogen action. Physiol Rev. 2001 Oct;81(4):1535-65. 
45. Prossnitz ER, Arterburn JB, Sklar LA. GPR30: A G protein-coupled receptor for estrogen. Mol Cell Endocrinol. 2007 Feb;265-266:138-42.

46. Mauvais-Jarvis F, Clegg DJ, Hevener AL. The role of estrogens in control of energy balance and glucose homeostasis. Endocr Rev. 2013 Jun;34(3):309-38.

47. Klein SL, Flanagan KL. Sex differences in immune responses. Nat Rev Immunol. 2016 Oct;16(10):626-38.

48. Wang J, Syrett CM, Kramer MC, Basu A, Atchison ML, Anguera MC. Unusual maintenance of $X$ chromosome inactivation predisposes female lymphocytes for increased expression from the inactive X. Proc Natl Acad Sci U S A. 2016 Apr 5;113(14):E2029-38.

49. Ngo ST, Steyn FJ, McCombe PA. Gender differences in autoimmune disease. Front Neuroendocrinol. 2014 Aug;35(3):347-69.

50. Eaton WW, Rose NR, Kalaydjian A, Pedersen MG, Mortensen PB. Epidemiology of autoimmune diseases in Denmark. J Autoimmun. 2007 Aug;29(1):1-9.

51. Markle JGM, Frank DN, Mortin-Toth S, Robertson CE, Feazel LM, Rolle-Kampczyk U, et al. Sex differences in the gut microbiome drive hormone-dependent regulation of autoimmunity. Science. 2013 Mar 1;339(6123):1084-8.

52. Cook IF. Sexual dimorphism of humoral immunity with human vaccines. Vaccine. $2008 \mathrm{Jul}$ 4;26(29-30):3551-5.

53. Mauvais-Jarvis F, Berthold HK, Campesi I, Carrero J-J, Dakal S, Franconi F, et al. Sex- and Gender-Based Pharmacological Response to Drugs. Pharmacol Rev. 2021 Apr;73(2):730-62.

54. Soldin OP, Mattison DR. Sex differences in pharmacokinetics and pharmacodynamics. Clin Pharmacokinet. 2009;48(3):143-57.

55. Anderson GD. Pregnancy-induced changes in pharmacokinetics: a mechanistic-based approach. Clin Pharmacokinet. 2005;44(10):989-1008.

56. Harward JL, Clinard VB, Jiroutek MR, Lingerfeldt BH, Muzyk AJ. Impact of a US Food and Drug Administration Drug Safety Communication on Zolpidem Dosing: An Observational Retrospective Cohort. Prim Care Companion CNS Disord [Internet]. 2015 Mar 5;17(2). Available from: http://dx.doi.org/10.4088/PCC.14m01728

57. Greenblatt DJ, Harmatz JS, Singh NN, Steinberg F, Roth T, Moline ML, et al. Gender differences in pharmacokinetics and pharmacodynamics of zolpidem following sublingual administration. J Clin Pharmacol. 2014 Mar;54(3):282-90.

58. Nelson DR, Nebert DW. Cytochrome P450 (CYP) Gene Superfamily. In: eLS [Internet]. Chichester, UK: John Wiley \& Sons, Ltd; 2018. p. 1-19. Available from: https://onlinelibrary.wiley.com/doi/10.1002/9780470015902.a0005667.pub3

59. Anderson GD. Sex and racial differences in pharmacological response: where is the evidence? Pharmacogenetics, pharmacokinetics, and pharmacodynamics. J Womens Health . 2005 Jan;14(1):19-29. 
60. Zhang LH, Rodriguez H, Ohno S, Miller WL. Serine phosphorylation of human P450c17 increases 17,20-lyase activity: implications for adrenarche and the polycystic ovary syndrome. Proc Natl Acad Sci U S A. 1995 Nov 7;92(23):10619-23.

61. van Anders SM, Steiger J, Goldey KL. Effects of gendered behavior on testosterone in women and men. Proc Natl Acad Sci U S A. 2015 Nov 10;112(45):13805-10.

62. Bellenghi M, Puglisi R, Pontecorvi G, De Feo A, Carè A, Mattia G. Sex and Gender Disparities in Melanoma. Cancers [Internet]. 2020 Jul 7;12(7). Available from: http://dx.doi.org/10.3390/cancers12071819

63. Paddock LE, Lu SE, Bandera EV, Rhoads GG, Fine J, Paine S, et al. Skin self-examination and long-term melanoma survival. Melanoma Res. 2016 Aug;26(4):401-8.

64. Campesi I, Romani A, Franconi F. The Sex-Gender Effects in the Road to Tailored Botanicals. Nutrients [Internet]. 2019 Jul 17;11(7). Available from:

http://dx.doi.org/10.3390/nu11071637

65. Kristoffersen AE, Stub T, Salamonsen A, Musial F, Hamberg K. Gender differences in prevalence and associations for use of CAM in a large population study. BMC Complement Altern Med. 2014 Dec 3;14:463.

66. Kim YS, Unno T, Kim BY, Park MS. Sex Differences in Gut Microbiota. World J Mens Health. 2020 Jan;38(1):48-60.

67. Carmody RN, Turnbaugh PJ. Host-microbial interactions in the metabolism of therapeutic and diet-derived xenobiotics. J Clin Invest. 2014 Oct;124(10):4173-81.

68. Higgins ST, Kurti AN, Redner R, White TJ, Gaalema DE, Roberts ME, et al. A literature review on prevalence of gender differences and intersections with other vulnerabilities to tobacco use in the United States, 2004-2014. Prev Med. 2015 Nov;80:89-100.

69. Zevin S, Benowitz NL. Drug interactions with tobacco smoking. An update. Clin Pharmacokinet. 1999 Jun;36(6):425-38.

70. Kroon LA. Drug interactions with smoking. Am J Health Syst Pharm. 2007 Sep 15;64(18):1917-21.

71. Burkman R, Schlesselman JJ, Zieman M. Safety concerns and health benefits associated with oral contraception. Am J Obstet Gynecol. 2004 Apr;190(4 Suppl):S5-22.

72. Schiff I, Bell WR, Davis V, Kessler CM, Meyers C, Nakajima S, et al. Oral contraceptives and smoking, current considerations: recommendations of a consensus panel. Am J Obstet Gynecol. 1999 Jun;180(6 Pt 2):S383-4.

73. Harmful Interactions [Internet]. [cited 2021 Sep 1]. Available from: https://www.niaaa.nih.gov/publications/brochures-and-fact-sheets/harmful-interactions-mixin g-alcohol-with-medicines

74. Slade T, Chapman C, Swift W, Keyes K, Tonks Z, Teesson M. Birth cohort trends in the global epidemiology of alcohol use and alcohol-related harms in men and women: systematic review and metaregression. BMJ Open. 2016 Oct 24;6(10):e011827. 
75. Kezer CA, Simonetto DA, Shah VH. Sex Differences in Alcohol Consumption and Alcohol-Associated Liver Disease. Mayo Clin Proc. 2021 Apr;96(4):1006-16.

76. Nosengo N. Can you teach old drugs new tricks? [Internet]. 2016 [cited 2020 Nov 18]. Available from: http://www.nature.com/news/can-you-teach-old-drugs-new-tricks-1.20091

77. DiMasi JA, Grabowski HG, Hansen RW. Innovation in the pharmaceutical industry: New estimates of R\&D costs. J Health Econ. 2016 May;47:20-33.

78. Corsello SM, Nagari RT, Spangler RD, Rossen J, Kocak M, Bryan JG, et al. Discovering the anticancer potential of non-oncology drugs by systematic viability profiling. Nature Cancer. 2020 Feb 1;1(2):235-48.

79. Alaimo S, Pulvirenti A. Network-Based Drug Repositioning: Approaches, Resources, and Research Directions. Methods Mol Biol. 2019;1903:97-113.

80. Pulley JM, Rhoads JP, Jerome RN, Challa AP, Erreger KB, Joly MM, et al. Using What We Already Have: Uncovering New Drug Repurposing Strategies in Existing Omics Data. Annu Rev Pharmacol Toxicol. 2020 Jan 6;60:333-52.

81. Hodos RA, Kidd BA, Shameer K, Readhead BP, Dudley JT. In silico methods for drug repurposing and pharmacology. Wiley Interdiscip Rev Syst Biol Med. 2016 May;8(3):186-210.

82. Rebholz-Schuhmann D, Oellrich A, Hoehndorf R. Text-mining solutions for biomedical research: enabling integrative biology. Nat Rev Genet. 2012 Dec;13(12):829-39.

83. Evidence Summary: Aspirin Use to Prevent Cancer: Aspirin Use to Prevent Cardiovascular Disease and Colorectal Cancer: Preventive Medication [Internet]. [cited 2021 Sep 9]. Available from: https://www.uspreventiveservicestaskforce.org/uspstf/document/evidence-summary-aspirinto-prevent-cancer/aspirin-to-prevent-cardiovascular-disease-and-cancer

84. Pinzi L, Rastelli G. Molecular Docking: Shifting Paradigms in Drug Discovery. Int J Mol Sci [Internet]. 2019 Sep 4;20(18). Available from: http://dx.doi.org/10.3390/ijms20184331

85. Dong D, Zhijian X, Wu Z, Shaoliang P. Parallelization of Molecular Docking: A Review. Curr Top Med Chem. 2018 May 1;18(12):1015-28.

86. Vilar S, Hripcsak G. The role of drug profiles as similarity metrics: applications to repurposing, adverse effects detection and drug-drug interactions. Brief Bioinform. 2017 Jul $1 ; 18(4): 670-81$.

87. Haugh IM, Preston AK, Kivelevitch DN, Menter AM. Risankizumab: an anti-IL-23 antibody for the treatment of psoriasis. Drug Des Devel Ther. 2018 Nov 12;12:3879-83.

88. Wang F, Lei X, Wu F-X. A review of drug repositioning based chemical-induced cell line expression data. Curr Med Chem [Internet]. 2018 Nov 1; Available from:

http://dx.doi.org/10.2174/0929867325666181101115801

89. Chen B, Ma L, Paik H, Sirota M, Wei W, Chua M-S, et al. Reversal of cancer gene expression correlates with drug efficacy and reveals therapeutic targets. Nat Commun. 2017 Jul 12;8:16022. 
90. Zhou X, Wang M, Katsyv I, Irie H, Zhang B. EMUDRA: Ensemble of Multiple Drug Repositioning Approaches to improve prediction accuracy. Bioinformatics. 2018 Sep 15;34(18):3151-9.

91. Duan Y, Evans DS, Miller RA, Schork NJ, Cummings SR, Girke T. signatureSearch: environment for gene expression signature searching and functional interpretation. Nucleic Acids Res. 2020 Dec 2;48(21):e124.

92. Iorio F, Saez-Rodriguez J, di Bernardo D. Network based elucidation of drug response: from modulators to targets. BMC Syst Biol. 2013 Dec 13;7:139.

93. Morselli Gysi D, do Valle Í, Zitnik M, Ameli A, Gan X, Varol O, et al. Network medicine framework for identifying drug-repurposing opportunities for COVID-19. Proc Natl Acad Sci U S A [Internet]. 2021 May 11;118(19). Available from:

http://dx.doi.org/10.1073/pnas.2025581118

94. Misselbeck K, Parolo S, Lorenzini F, Savoca V, Leonardelli L, Bora P, et al. A network-based approach to identify deregulated pathways and drug effects in metabolic syndrome. Nat Commun. 2019 Nov 18;10(1):5215.

95. Lotfi Shahreza M, Ghadiri N, Mousavi SR, Varshosaz J, Green JR. A review of network-based approaches to drug repositioning. Brief Bioinform. 2018 Sep 28;19(5):878-92.

96. Parca L, Truglio M, Biagini T, Castellana S, Petrizzelli F, Capocefalo D, et al. Pyntacle: a parallel computing-enabled framework for large-scale network biology analysis.

Gigascience [Internet]. 2020 Oct 21;9(10). Available from:

http://dx.doi.org/10.1093/gigascience/giaa115

97. Fang J, Zhang P, Zhou Y, Chiang C-W, Tan J, Hou Y, et al. Endophenotype-based in silico network medicine discovery combined with insurance record data mining identifies sildenafil as a candidate drug for Alzheimer's disease. Nature Aging. 2021 Dec 6;1-14.

98. Avram S, Bologa CG, Holmes J, Bocci G, Wilson TB, Nguyen D-T, et al. DrugCentral 2021 supports drug discovery and repositioning. Nucleic Acids Res. 2021 Jan 8;49(D1):D1160-9.

99. Center for Drug Evaluation, Research. Questions and Answers on FDA's Adverse Event Reporting System (FAERS) [Internet]. [cited 2021 Nov 9]. Available from:

https://www.fda.gov/drugs/surveillance/questions-and-answers-fdas-adverse-event-reportin g-system-faers

100. Kfoury N, Qi Z, Prager BC, Wilkinson MN, Broestl L, Berrett KC, et al. Brd4-bound enhancers drive cell-intrinsic sex differences in glioblastoma. Proc Natl Acad Sci U S A [Internet]. 2021 Apr 20;118(16). Available from: http://dx.doi.org/10.1073/pnas.2017148118

101. Bowen RL, Perry G, Xiong C, Smith MA, Atwood CS. A clinical study of lupron depot in the treatment of women with Alzheimer's disease: preservation of cognitive function in patients taking an acetylcholinesterase inhibitor and treated with high dose lupron over 48 weeks. J Alzheimers Dis. 2015;44(2):549-60.

102. Bowen RL, Butler T, Atwood CS. [No title] [Internet]. American Society of Clinical Oncology; [cited 2021 Aug 16]. Available from: 
https://ascopubs.org/doi/10.1200/JCO.2015.66.3997?url_ver=Z39.88-2003\&rfr_id=ori:rid:cr ossref.org\&rfr_dat=cr_pub\%20\%200pubmed

103. Padi M, Quackenbush J. Detecting phenotype-driven transitions in regulatory network structure. NPJ Syst Biol Appl. 2018 Apr 19;4:16.

104. Bernabeu E, Canela-Xandri O, Rawlik K, Talenti A, Prendergast J, Tenesa A. Sexual differences in genetic architecture in UK Biobank [Internet]. bioRxiv. 2020. p. 2020.07.20.211813. Available from: https://www.biorxiv.org/content/10.1101/2020.07.20.211813v1

105. Saunders CL, Massou E. Using UK Biobank for sexual minority health research. J Comp Eff Res. 2021 Sep;10(13):1001-10.

106. Flynn E, Chang A, Altman RB. Large-Scale Labeling and Assessment of Sex Bias in Publicly Available Expression Data [Internet]. bioRxiv. 2020 [cited 2021 Apr 6]. p. 2020.10.26.356287. Available from: https://www.biorxiv.org/content/10.1101/2020.10.26.356287v1

107. Woitowich NC, Beery A, Woodruff T. A 10-year follow-up study of sex inclusion in the biological sciences. Elife [Internet]. 2020 Jun 9;9. Available from: http://dx.doi.org/10.7554/eLife.56344

108. Itoh Y, Arnold AP. Are females more variable than males in gene expression? Meta-analysis of microarray datasets. Biol Sex Differ. 2015 Oct 29;6:18.

109. Vilar S, Tatonetti NP, Hripcsak G. 3D pharmacophoric similarity improves multi adverse drug event identification in pharmacovigilance. Sci Rep. 2015 Mar 6;5:8809.

110. Wise AL, Gyi L, Manolio TA. eXclusion: toward integrating the $X$ chromosome in genome-wide association analyses. Am J Hum Genet. 2013 May 2;92(5):643-7.

111. Webster TH, Couse M, Grande BM, Karlins E, Phung TN, Richmond PA, et al. Identifying, understanding, and correcting technical artifacts on the sex chromosomes in next-generation sequencing data. Gigascience [Internet]. 2019 Jul 1;8(7). Available from: http://dx.doi.org/10.1093/gigascience/giz074

112. Olney KC, Brotman SM, Andrews JP, Valverde-Vesling VA, Wilson MA. Reference genome and transcriptome informed by the sex chromosome complement of the sample increase ability to detect sex differences in gene expression from RNA-Seq data. Biol Sex Differ. 2020 Jul 21;11(1):42.

113. Gamazon ER, Im HK, O'Donnell PH, Ziliak D, Stark AL, Cox NJ, et al. Comprehensive evaluation of the contribution of $X$ chromosome genes to platinum sensitivity. Mol Cancer Ther. 2011 Mar;10(3):472-80.

114. Molaro A, Malik HS. Culture shock. Elife [Internet]. 2017 Dec 18;6. Available from: http://dx.doi.org/10.7554/eLife.33312

115. Bansal P, Ahern DT, Kondaveeti Y, Qiu CW, Pinter SF. Contiguous erosion of the inactive $X$ in human pluripotency concludes with global DNA hypomethylation. Cell Rep. 2021 Jun 8;35(10):109215. 
116. Mittwoch U. Sex-determining mechanisms in animals. Trends Ecol Evol. 1996 Feb;11(2):63-7.

117. Cline TW, Meyer BJ. VIVE LA DIFFÉRENCE: Males vs females in flies vs worms. Annu Rev Genet. 1996 Dec;30(1):637-702.

118. Liew WC, Orbán L. Zebrafish sex: a complicated affair. Brief Funct Genomics. 2014 Mar;13(2):172-87.

119. Gasque G, Conway S, Huang J, Rao Y, Vosshall LB. Small molecule drug screening in Drosophila identifies the $5 \mathrm{HT} 2 \mathrm{~A}$ receptor as a feeding modulation target. Sci Rep. 2013;3:srep02120.

120. O'Reilly LP, Luke CJ, Perlmutter DH, Silverman GA, Pak SC. C. elegans in high-throughput drug discovery. Adv Drug Deliv Rev. 2014 Apr;69-70:247-53.

121. Parng C, Seng WL, Semino C, McGrath P. Zebrafish: a preclinical model for drug screening. Assay Drug Dev Technol. 2002 Nov;1(1 Pt 1):41-8.

122. Lemaître $J-F$, Ronget $V$, Tidière $M$, Allainé $D$, Berger $V$, Cohas $A$, et al. Sex differences in adult lifespan and aging rates of mortality across wild mammals. Proc Natl Acad Sci U S A. 2020 Apr 14;117(15):8546-53.

123. Yuan R, Musters CJM, Zhu Y, Evans TR, Sun Y, Chesler EJ, et al. Genetic differences and longevity-related phenotypes influence lifespan and lifespan variation in a sex-specific manner in mice. Aging Cell. 2020 Nov;19(11):e13263.

124. Bell MR. Comparing Postnatal Development of Gonadal Hormones and Associated Social Behaviors in Rats, Mice, and Humans. Endocrinology. 2018 Jul 1;159(7):2596-613.

125. McEvoy CM, Murphy JM, Zhang L, Clotet-Freixas S, Mathews JA, An J, et al. Single-cell profiling of healthy human kidney reveals features of sex-based transcriptional programs and tissue-specific immunity [Internet]. bioRxiv. 2021 [cited 2021 Dec 19]. p. 2021.12.09.471943. Available from: https://www.biorxiv.org/content/10.1101/2021.12.09.471943v1

126. Miller LR, Marks C, Becker JB, Hurn PD, Chen W-J, Woodruff T, et al. Considering sex as a biological variable in preclinical research. FASEB J. 2017 Jan;31(1):29-34.

127. Tannenbaum C, Ellis RP, Eyssel F, Zou J, Schiebinger L. Sex and gender analysis improves science and engineering. Nature. 2019 Nov;575(7781):137-46.

128. Becker JB, Prendergast BJ, Liang JW. Female rats are not more variable than male rats: a meta-analysis of neuroscience studies. Biol Sex Differ. 2016 Jul 26;7:34.

129. Prendergast BJ, Onishi KG, Zucker I. Female mice liberated for inclusion in neuroscience and biomedical research. Neurosci Biobehav Rev. 2014 Mar;40:1-5.

130. Buch T, Moos K, Ferreira FM, Fröhlich H, Gebhard C, Tresch A. Benefits of a factorial design focusing on inclusion of female and male animals in one experiment. J Mol Med . 2019 Jun;97(6):871-7.

131. Beery AK. Inclusion of females does not increase variability in rodent research studies. 
Curr Opin Behav Sci. 2018 Oct;23:143-9.

132. Garcia-Sifuentes $Y$, Maney DL. Reporting and misreporting of sex differences in the biological sciences. Elife. 2021 Nov 2;10:e70817.

133. Parker KL, Rice DA, Lala DS, Ikeda Y, Luo X, Wong M, et al. Steroidogenic factor 1: an essential mediator of endocrine development. Recent Prog Horm Res. 2002;57:19-36.

134. Burgoyne PS, Arnold AP. A primer on the use of mouse models for identifying direct sex chromosome effects that cause sex differences in non-gonadal tissues. Biol Sex Differ. 2016 Dec 13;7:68.

135. Walker VR, Korach KS. Estrogen receptor knockout mice as a model for endocrine research. ILAR J. 2004;45(4):455-61.

136. Kerkhofs S, Denayer S, Haelens A, Claessens F. Androgen receptor knockout and knock-in mouse models. J Mol Endocrinol. 2009 Jan;42(1):11-7.

137. De Vries GJ, Rissman EF, Simerly RB, Yang L-Y, Scordalakes EM, Auger CJ, et al. A model system for study of sex chromosome effects on sexually dimorphic neural and behavioral traits. J Neurosci. 2002 Oct 15;22(20):9005-14.

138. Arnold AP, Chen X. What does the "four core genotypes" mouse model tell us about sex differences in the brain and other tissues? Front Neuroendocrinol. 2009 Jan;30(1):1-9.

139. Hägg S, Jylhävä J. Sex differences in biological aging with a focus on human studies. Elife [Internet]. 2021 May 13;10. Available from: http://dx.doi.org/10.7554/eLife.63425

140. Melloni C, Berger JS, Wang TY, Gunes F, Stebbins A, Pieper KS, et al. Representation of women in randomized clinical trials of cardiovascular disease prevention. Circ Cardiovasc Qual Outcomes. 2010 Mar;3(2):135-42.

141. Yoon DY, Mansukhani NA, Stubbs VC, Helenowski IB, Woodruff TK, Kibbe MR. Sex bias exists in basic science and translational surgical research. Surgery. 2014 Sep;156(3):508-16.

142. Clayton JA. Studying both sexes: a guiding principle for biomedicine. FASEB J. 2016 Feb;30(2):519-24.

143. Jeong S, Kim J, Park W, Jeon H, Kim N. SEXCMD: Development and validation of sex marker sequences for whole-exome/genome and RNA sequencing. PLoS One. 2017 Sep 8;12(9):e0184087.

144. Ryaboshapkina M, Hammar M. Tissue-specific genes as an underutilized resource in drug discovery. Sci Rep. 2019 May 10;9(1):7233.

145. Genomic Intelligence for Pharma with Mastermind [Internet]. 2020 [cited 2021 Oct 19]. Available from: https://www.genomenon.com/pharma/

146. Sheils T, Mathias SL, Siramshetty VB, Bocci G, Bologa CG, Yang JJ, et al. How to Illuminate the Druggable Genome Using Pharos. Curr Protoc Bioinformatics. 2020 Mar;69(1):e92. 
147. Iwata H, Sawada R, Mizutani S, Yamanishi Y. Systematic drug repositioning for a wide range of diseases with integrative analyses of phenotypic and molecular data. J Chem Inf Model. 2015 Feb 23;55(2):446-59.

148. Chupakhin V, Marcou G, Baskin I, Varnek A, Rognan D. Predicting ligand binding modes from neural networks trained on protein-ligand interaction fingerprints. J Chem Inf Model. 2013 Apr 22;53(4):763-72.

149. Napolitano F, Zhao Y, Moreira VM, Tagliaferri R, Kere J, D’Amato M, et al. Drug repositioning: a machine-learning approach through data integration. J Cheminform. 2013 Jun 22;5(1):30.

150. Vilar S, Ryan PB, Madigan D, Stang PE, Schuemie MJ, Friedman C, et al. Similarity-based modeling applied to signal detection in pharmacovigilance. CPT Pharmacometrics Syst Pharmacol. 2014 Sep 24;3:e137.

151. Cao R, Wang Y. Predicting Molecular Targets for Small-Molecule Drugs with a Ligand-Based Interaction Fingerprint Approach. ChemMedChem. 2016 Jun 20;11(12):1352-61.

152. Cheng F, Li W, Wu Z, Wang X, Zhang C, Li J, et al. Prediction of polypharmacological profiles of drugs by the integration of chemical, side effect, and therapeutic space. J Chem Inf Model. 2013 Apr 22;53(4):753-62.

153. Chan J, Wang X, Turner JA, Baldwin NE, Gu J. Breaking the paradigm: Dr Insight empowers signature-free, enhanced drug repurposing. Bioinformatics. 2019 Aug 15;35(16):2818-26.

154. Sanseau P, Agarwal P, Barnes MR, Pastinen T, Richards JB, Cardon LR, et al. Use of genome-wide association studies for drug repositioning. Nat Biotechnol. 2012 Apr 10;30(4):317-20.

155. Grover MP, Ballouz S, Mohanasundaram KA, George RA, Goscinski A, Crowley TM, et al. Novel therapeutics for coronary artery disease from genome-wide association study data. BMC Med Genomics. 2015 May 29;8 Suppl 2:S1.

156. Rivas-Barragan D, Mubeen S, Guim Bernat F, Hofmann-Apitius M, Domingo-Fernández D. Drug2ways: Reasoning over causal paths in biological networks for drug discovery. PLoS Comput Biol. 2020 Dec;16(12):e1008464.

157. Greene CS, Krishnan A, Wong AK, Ricciotti E, Zelaya RA, Himmelstein DS, et al. Understanding multicellular function and disease with human tissue-specific networks. Nat Genet. 2015 Jun;47(6):569-76.

158. Subramanian A, Narayan R, Corsello SM, Peck DD, Natoli TE, Lu X, et al. A Next Generation Connectivity Map: L1000 Platform and the First 1,000,000 Profiles. Cell. 2017 Nov 30;171(6):1437-52.e17.

159. Iljin K, Ketola K, Vainio P, Halonen P, Kohonen P, Fey V, et al. High-throughput cell-based screening of 4910 known drugs and drug-like small molecules identifies disulfiram as an inhibitor of prostate cancer cell growth. Clin Cancer Res. 2009 Oct 1;15(19):6070-8. 
160. Shen M, Asawa R, Zhang Y-Q, Cunningham E, Sun H, Tropsha A, et al. Quantitative high-throughput phenotypic screening of pediatric cancer cell lines identifies multiple opportunities for drug repurposing. Oncotarget. 2018 Jan 12;9(4):4758-72.

161. Martinez Molina D, Jafari R, Ignatushchenko M, Seki T, Larsson EA, Dan C, et al. Monitoring drug target engagement in cells and tissues using the cellular thermal shift assay. Science. 2013 Jul 5;341(6141):84-7.

162. Miettinen TP, Björklund $\mathrm{M}$. NQO2 is a reactive oxygen species generating off-target for acetaminophen. Mol Pharm. 2014 Dec 1;11(12):4395-404.

163. De Vries GJ, Panzica GC. Sexual differentiation of central vasopressin and vasotocin systems in vertebrates: different mechanisms, similar endpoints. Neuroscience. 2006;138(3):947-55. 